\title{
Longitudinal analyses reveal immunological misfiring in severe COVID-19
}

https://doi.org/10.1038/s41586-020-2588-y

Received: 23 June 2020

Accepted: 21 July 2020

Published online: 27 July 2020

Check for updates

\begin{abstract}
Carolina Lucas $^{1,17}$, Patrick Wong ${ }^{1,17}$, Jon Klein ${ }^{1,17}$, Tiago B. R. Castro ${ }^{2,17}$, Julio Silva', Maria Sundaram ${ }^{3}$, Mallory K. Ellingson ${ }^{3}$, Tianyang Mao', Ji Eun Oh', Benjamin Israelow ${ }^{1,4}$, Takehiro Takahashi', Maria Tokuyama', Peiwen Lu', Arvind Venkataraman', Annsea Park', Subhasis Mohanty ${ }^{4}$, Haowei Wang ${ }^{4}$, Anne L. Wyllie ${ }^{3}$, Chantal B. F. Vogels ${ }^{3}$, Rebecca Earnest ${ }^{3}$, Sarah Lapidus ${ }^{3}$, Isabel M. Ott ${ }^{3}$, Adam J. Moore ${ }^{3}$, M. Catherine Muenker ${ }^{3}$, John B. Fournier ${ }^{4}$, Melissa Campbell ${ }^{4}$, Camila D. Odio ${ }^{4}$, Arnau Casanovas-Massana ${ }^{3}$, Yale IMPACT Team*, Roy Herbst ${ }^{5}$, Albert C. Shaw ${ }^{4}$, Ruslan Medzhitov ${ }^{1,6}$, Wade L. Schulz ${ }^{7,8}$, Nathan D. Grubaugh ${ }^{3}$, Charles Dela Cruz ${ }^{9}$, Shelli Farhadian ${ }^{4}$, Albert I. $\mathrm{Ko}^{3,4}$, Saad B. Omer ${ }^{3,4,10}$ \& Akiko Iwasaki ${ }^{1,6 凶}$
\end{abstract}

\begin{abstract}
Recent studies have provided insights into the pathogenesis of coronavirus disease 2019 (COVID-19) ${ }^{1-4}$. However, the longitudinal immunological correlates of disease outcome remain unclear. Here we serially analysed immune responses in 113 patients with moderate or severe COVID-19. Immune profiling revealed an overall increase in innate cell lineages, with a concomitant reduction in $T$ cell number. An early elevation in cytokine levels was associated with worse disease outcomes. Following an early increase in cytokines, patients with moderate COVID-19 displayed a progressive reduction in type 1 (antiviral) and type 3 (antifungal) responses. By contrast, patients with severe COVID-19 maintained these elevated responses throughout the course of the disease. Moreover, severe COVID-19 was accompanied by an increase in multiple type 2 (anti-helminths) effectors, including interleukin-5 (IL-5), IL-13, immunoglobulin E and eosinophils. Unsupervised clustering analysis identified four immune signatures, representing growth factors (A), type-2/3 cytokines (B), mixed type-1/2/3 cytokines (C), and chemokines (D) that correlated with three distinct disease trajectories. The immune profiles of patients who recovered from moderate COVID-19 were enriched in tissue reparative growth factor signature $A$, whereas the profiles of those with who developed severe disease had elevated levels of all four signatures. Thus, we have identified a maladapted immune response profile associated with severe COVID-19 and poor clinical outcome, as well as early immune signatures that correlate with divergent disease trajectories.
\end{abstract}

COVID-19 is caused by severe acute respiratory syndrome coronavirus 2 (SARS-CoV-2), a highly infectious virus that exploits angiotensin-converting enzyme $2(\mathrm{ACE} 2)^{5,6}$ as a cell entry receptor. The clinical presentation of COVID-19 involves a broad range of symptoms and disease trajectories. Understanding the nature of the immune response that leads to recovery over severe disease is key to developing effective treatments for COVID-19. Coronaviruses, including Severe Acute Respiratory Syndrome (SARS-CoV) and Middle Eastern Respiratory Syndrome (MERS), typically induce strong inflammatory responses and associated lymphopenia ${ }^{78}$. Studies of patients with COVID-19 have reported increases in inflammatory monocytes and neutrophils, and a sharp decrease in lymphocytes ${ }^{1-4}$, and an inflammatory milieu containing IL-1 $\beta$, IL-6, and TNF (previously known as TNF $\alpha$ ) in severe disease $\mathrm{e}^{1,2,4,9,10}$. Despite these analyses, the dynamics of the immune response during the course of SARS-CoV-2 infection and its association with clinical trajectory remain unclear.

Immune responses against pathogens are divided roughly into three types $^{11-13}$. Type 1 immunity, characterized by responses that depend on the transcription factor T-bet (also known as TBX21) and expression of interferon- $\gamma$ (IFN $\gamma)$, is generated against intracellular pathogens such as viruses. In type 1 immunity, pathogen clearance is mediated through effector cells including group 1 innate lymphocytes (ILC1), natural killer (NK) cells, cytotoxic T lymphocytes, and T helper $1\left(T_{H} 1\right)$ cells. Type 2 immunity, which relies on the GATA3 transcription factor, mediates defence against helminths through effector molecules such as IL-4, IL-5, IL-13, and IgE that work to expel these pathogens through the concerted action of epithelial cells, mast cells, eosinophils, and basophils. Type 3 immunity, which is orchestrated by the ROR $\gamma t$-induced cytokines IL-17

${ }^{1}$ Department of Immunobiology, Yale University School of Medicine, New Haven, CT, USA. ${ }^{2}$ Laboratory of Mucosal Immunology, The Rockefeller University, New York, NY, USA. ${ }^{3}$ Department of Epidemiology of Microbial Diseases, Yale School of Public Health, New Haven, CT, USA. ${ }^{4}$ Department of Medicine, Section of Infectious Diseases, Yale University School of Medicine, New Haven, CT, USA. ${ }^{5}$ Yale University School of Medicine, Yale Cancer Center, and Smilow Cancer Hospital, New Haven, CT, USA. ${ }^{6} \mathrm{Howard}$ Hughes Medical Institute, Chevy Chase, MD, USA. ${ }^{7}$ Department of Laboratory Medicine, Yale University School of Medicine, New Haven, CT, USA. ${ }^{8}$ Center for Outcomes Research and Evaluation, Yale-New Haven Hospital, New Haven, CT, USA. ${ }^{9}$ Department of

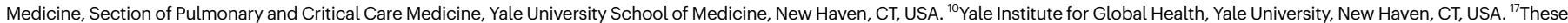
authors contributed equally: Carolina Lucas, Patrick Wong, Jon Klein, Tiago B. R. Castro. *A list of authors and their affiliations appears at the end of the paper. ${ }^{\circledR} \mathrm{e}-\mathrm{mail}$ : akiko.iwasaki@yale.edu 
and IL-22 secreted by ILC 3 and $\mathrm{T}_{\mathrm{H}} 17$ cells, is mounted against fungi and extracellular bacteria to elicit neutrophil-dependent clearance. We have focused on the longitudinal analysis of these three types of immune response in patients with COVID-19 and identified correlations between distinct immune phenotypes and disease course.

\section{Immunological features of COVID-19}

One hundred and thirteen patients with COVID-19 who were admitted to Yale New Haven Hospital (YNHH) between 18 March 2020 and 27 May 2020 were recruited to the Yale IMPACT (Implementing Medical and Public Health Action Against Coronavirus CT) study. We assessed viral RNA load (quantified by quantitative PCR with reverse transcription (RT-qPCR) using nasopharyngeal swabs); levels of plasma cytokines and chemokines; and leukocyte profiles (by flow cytometry using freshly isolated peripheral blood mononuclear cells; PBMCs). We performed 253 collections and follow-up measurements on the patient cohort with a range of one to seven longitudinal time-points that occurred 3-51 days after the onset of symptoms. In parallel, we enrolled 108 volunteer healthcare workers (HCWs), whose samples served as healthy controls (SARS-CoV-2-negative by RT-qPCR and serology).

Basic demographic information stratified by disease severity is provided in Extended Data Table 1 and detailed in Supplementary Table 1. Patients who had been admitted to YNHH were stratified into moderate and severe disease groups on the basis of supplemental oxygen requirements and admission to the intensive care unit (ICU) (Fig. 1a). Among our cohort, patients who developed moderate or severe disease did not differ significantly with respect to age or sex. Body mass index (BMI) was generally higher among patients with severe disease, and extremes in BMI correlated with an increased relative risk (RR) of mortality (RR BMI $\geq 35: 1.62$ (95\% confidence interval (CI) 0.81-3.22)) (Extended Data Table 1, Extended Data Fig. 1a, b). Exposure to select therapeutic regimens of interest was assessed in patients with moderate or severe disease (Extended Data Fig. 1c.) Initial presenting symptoms demonstrated a preponderance of headache (54.55\%), fever (64.47\%), cough $(74.03 \%)$, and dyspnoea (67.09\%) with no significant difference in symptom presentation between patients with moderate disease and those who developed severe disease. Finally, mortality was significantly higher in patients who were admitted to the ICU than in those who were not $(27.27 \%$ versus $3.75 \% ; P<0.001)$ (Extended Data Table 1$)$.

We analysed PBMC and plasma samples from patients with moderate or severe COVID-19 and healthy HCW donors (Fig. 1a, gating strategy in Extended Data Fig. 9) by flow cytometry and ELISA to quantify leukocytes and soluble mediators, respectively. An unsupervised heat map constructed from the main innate and adaptive circulating immune cell types revealed marked changes in patients with COVID-19 compared to uninfected HCWs (Fig. 1b). As reported ${ }^{1-4}$, patients with COVID-19 presented with marked reductions in the number and frequency of both $\mathrm{CD}^{+}$and $\mathrm{CD} 8^{+} \mathrm{T}$ cells, even after normalizing for age as a possible confounder (Extended Data Fig. 1d). Granulocytes, such as neutrophils and eosinophils, are normally excluded from the PBMC fraction following density gradient separation. However, low-density granulocytes are found in the PBMC layer of peripheral blood collected from patients with inflammatory diseases ${ }^{14}$. In patients with COVID-19, increases in monocytes, low-density neutrophils and eosinophils correlated with the severity of disease (Fig. 2c, Extended Data Fig. 2a, b). In addition, patients showed increased activation of $\mathrm{T}$ cells and a reduction in expression of the human leukocyte antigen DR isotype (HLA-DR) by circulating monocytes ${ }^{1}$ (Extended Data Fig. 2c). A complete overview of PBMC subsets is presented in Extended Data Fig. 2.

To gain insights into key differences in cytokines, chemokines, and additional immune markers between patients with moderate and severe disease, we correlated the measurements of these soluble proteins across all sample collection time-points. (Fig. 1d). We observed a 'core COVID-19 signature' that was shared by both moderate and severe disease groups and was defined by the following inflammatory cytokines, which correlated positively with each other: IL-1 $\alpha$, IL-1 $\beta$, IL-17A, IL-12 p70, and IFN $\alpha$ (Fig. 1d). In patients with severe disease, we observed an additional inflammatory cluster defined by thrombopoietin (TPO), IL-33, IL-16, IL-21, IL-23, IFN $\lambda$, eotaxin and eotaxin 3 (Fig. 1d). Most of the cytokines linked to cytokine release syndrome (CRS), such as IL-1 $\alpha$, IL-1 1 , IL-6, IL-10, IL-18 and TNF, showed increased positive associations in patients with severe disease (Fig.1d-f, Extended Data Fig. 3). These data highlight broad inflammatory changes, involving concomitant release of type 1 , type 2 and type 3 cytokines, in patients with severe COVID-19.

\section{Longitudinal immune profiling of COVID-19}

Our data presented above, as well as previous single-cell transcriptome and flow-cytometry-based studies ${ }^{2,4,15-17}$, depicted overt innate and adaptive immune activation in patients with severe COVID-19. Longitudinal cytokine correlations, measured in terms of days from symptom onset (DfSO), indicated that major differences in immune phenotypes between moderate and severe disease were apparent after day 10 of infection (Fig. 2a). In the first 10 DfSO, patients with severe or moderate disease displayed similar correlation intensity and markers, including the overall core COVID-19 signature described above (Fig. 2a). After day 10 these markers declined steadily in patients with moderate disease. By contrast, patients with severe COVID-19 maintained elevated levels of these core signature makers. Notably, additional correlations between cytokines emerged in patients with severe disease following day 10 (Fig. 2a). These analyses strongly support the observation (Fig. 1) that TPO and IFN $\alpha$ associate strongly with IFN $\lambda$, IL-9, IL-18, IL-21, IL-23, and IL-33 (Fig. 2a). These observations indicate sharp differences in the expression of inflammatory markers along disease progression between patients who exhibit moderate versus severe symptoms of COVID-19.

Temporal analyses of PBMCs and soluble proteins in plasma, either by linear regression or grouped intervals, supported distinct courses in disease. IFN $\alpha$ levels were sustained at higher levels in patients with severe disease, but these declined in patients with moderate disease (Fig. 2b). Plasma levels of IFN $\lambda$ increased during the first week of symptoms in patients with severe disease, and remained elevated in later phases (Fig. 2b). In addition, inflammasome-induced cytokines, such as IL-1 $\beta$ and IL-18, were also higher in patients with severe disease than in patients with moderate disease at most time-points analysed (Fig. 2c). IL-1 receptor antagonist (IL-1Ra), which is induced by IL-1R signalling as a negative feedback regulator ${ }^{18}$, was also increased in patients with severe COVID-19 from day 10 of disease onset (Extended Data Fig. 4).

With respect to type 1 immunity, there was an increased number of monocytes at approximately 14 DfSO in patients with severe but not moderate COVID-19 (Fig. 2d). The innate cytokine IL-12, a key inducer of type-1 immunity ${ }^{11,12}$, displayed a similar pattern to IFN $\gamma$-increasing over time in patients with severe disease but declining steadily in those with moderate disease (Fig. 2d). Intracellular cytokine staining showed that $\mathrm{CD}^{+}$and $\mathrm{CD}^{+} \mathrm{T}$ cells from patients with moderate disease secreted comparable amounts of IFN $\gamma$ to those from patients with severe disease. Together with the severe $\mathrm{T}$ cell depletion seen in patients with severe disease (Fig. 1), our data suggest that secretion of IFN $\gamma$ by non-T cells (ILC1, NK cells), or non-circulating T cells in tissues was the primary contributor to the enhanced levels observed in patients with severe disease (Extended Data Fig. 5).

Type-2 immune markers continued to increase over time in patients with severe COVID-19, as indicated by the strong correlations observed at late time points for these patients (Fig. 2a). Eosinophils and levels of eotaxin-2 increased in patients with severe disease and remained higher than in patients with moderate disease (Fig. 2e). Type 2 innate immune cytokines, including thymic stromal lymphopoietin (TSLP) and IL-33, did not show significant differences between patients with severe and moderate disease (Fig. 2e). Levels of hallmark type 2 cytokines, including IL-5 (associated with eosinophilia) and IL-13 (Fig. 2e), were higher 
a

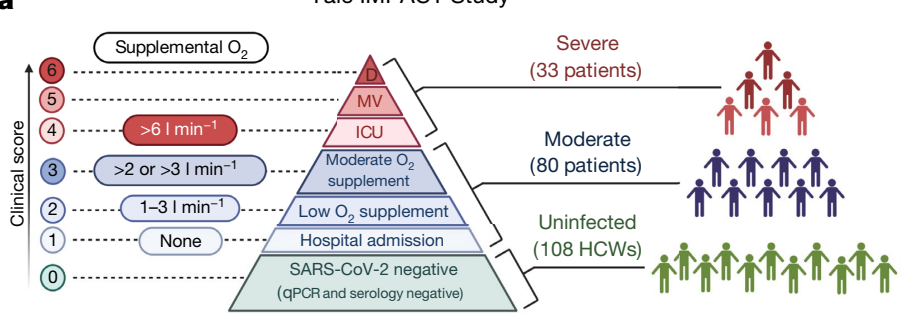

b

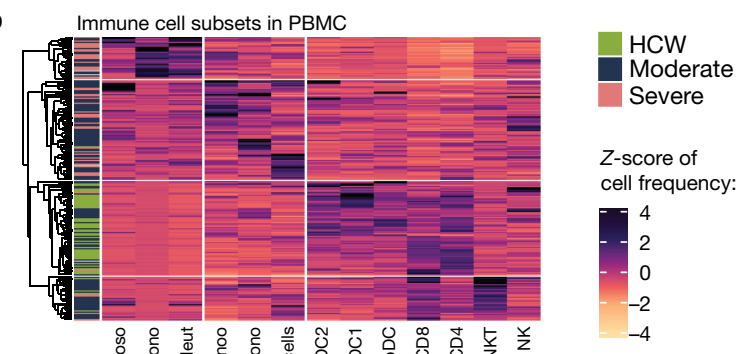

c
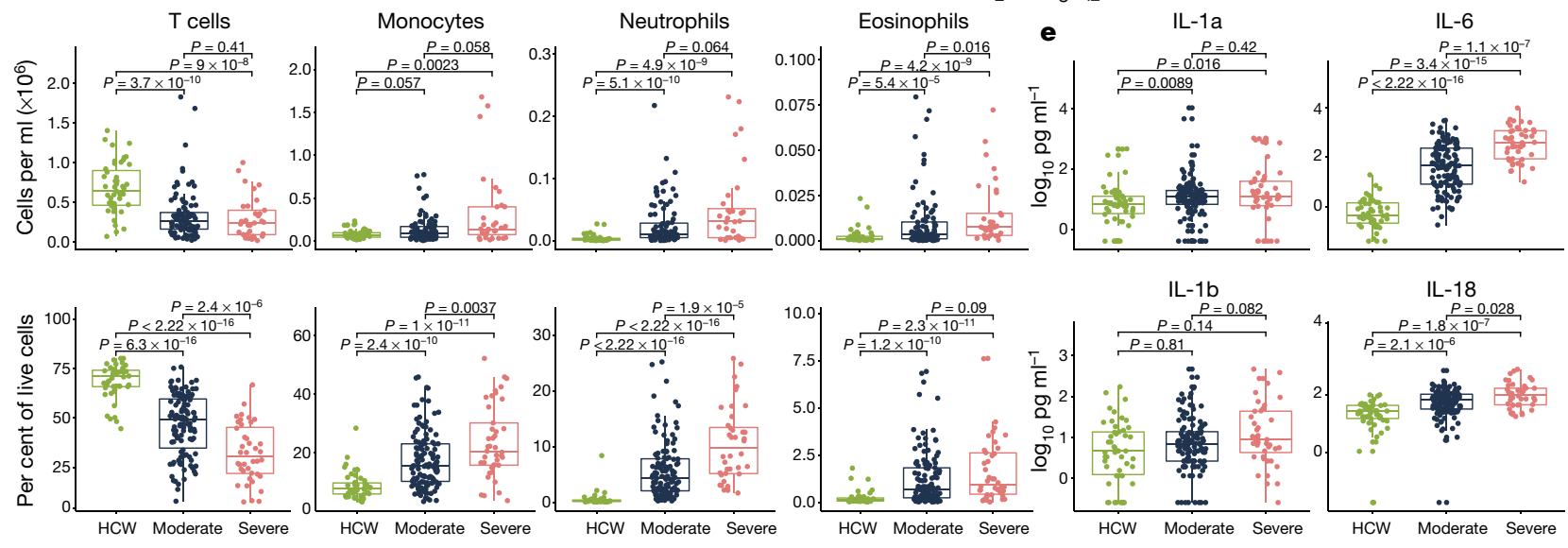

d

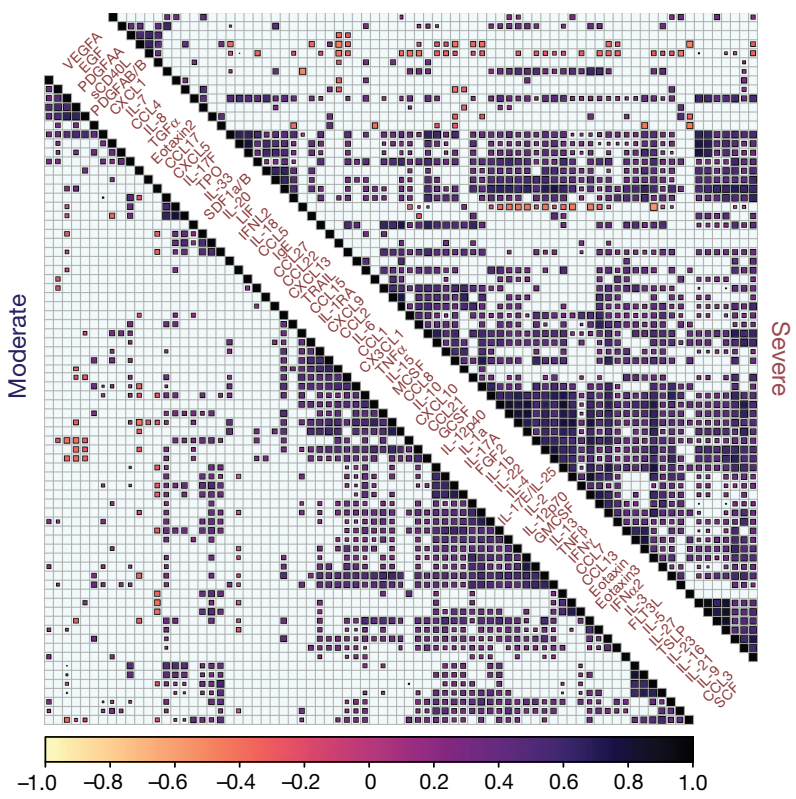

'
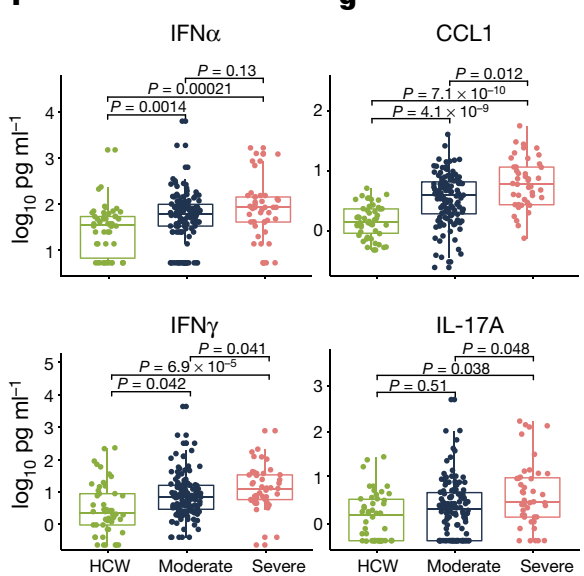

Fig. 1 Overview of immunological features in patients with COVID-19. a, Overview of cohort, including healthy donors (HCWs) and patients with moderate or severe COVID-19. Ordinal scores assigned according to clinical severity scale as described in Methods. D, deceased; ICU, intensive care unit; MV, mechanical ventilation. b, Heat map comparison of the major immune cell populations within PBMCs in patients with moderate $(n=121)$ or severe $(n=43)$ COVID-19, or HCSs $(n=43)$. $n$ values represent a separate time point per subject Subjects are arranged across rows, with each coloured unit indicating the relative distribution of an immune cell population normalized against the same population across all subjects. $K$-means clustering was used to arrange patients and measurements. Eoso, eosinophil; ncMono, non-classical monocyte; neut, neutrophil; cMono, classical monocyte; intMono, intermediate monocyte; DC2 and DC1, type 2 and 1 dendritic cells, respectively; pDC, plasmacytoid dendritic cell; T-CD8 and T-CD4, CD8 ${ }^{+}$and $C D 4^{+} \mathrm{T}$ cells,

in patients with severe disease than in those with moderate disease. By contrast, IL-4 levels were not significantly different. However, IL-4, similar to IL-5 and IL-13, showed an upward trend over the course of disease respectively; NKT, natural killer T cell; NK, natural killer cell.c, Immune cell subsets plotted as a concentration of millions of cells per millilitre of blood or as a percentage of live single cells. Each dot represents a separate time point per subject (HCW, $n=50$; moderate, $n=117$; severe, $n=40$ ). d, Correlation matrices across all time points of 71 cytokines from patient blood, comparing patients with moderate and severe disease. Only significant correlations $(<0.05)$ are represented as dots. Pearson's correlation coefficients from comparisons of cytokine measurements within the same patients are visualized by colour intensity. $\mathbf{e}-\mathbf{g}$, Quantification of prominent inflammatory cytokines (e), interferons type I and II (f), and CCL1 and IL-17 (g) presented as $\log _{10}$-transformed concentrations. Each dot represents a separate time point per subject (HCW, $n=50$; moderate, $n=117$; severe, $n=40$ ). Centre, median; box limits, first and third percentiles; whiskers, $1.5 \times$ interquartile range $(\mathrm{IQR})$. Significance determined by two-sided, Wilcoxon rank-sum test.

in patients with severe COVID-19 (Fig. 2e). The type 2 antibody isotype IgE was also higher in patients with severe diasease and continued to increase during the disease course (Fig. 2e). 

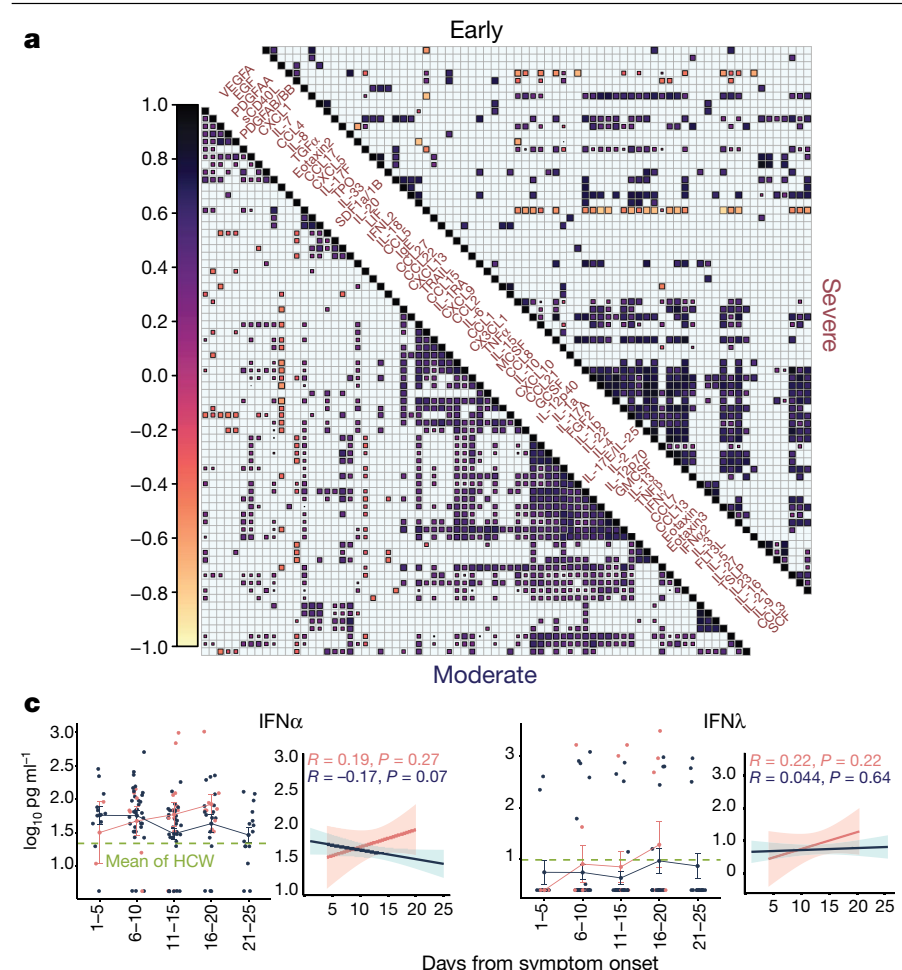

Days from symptom onset
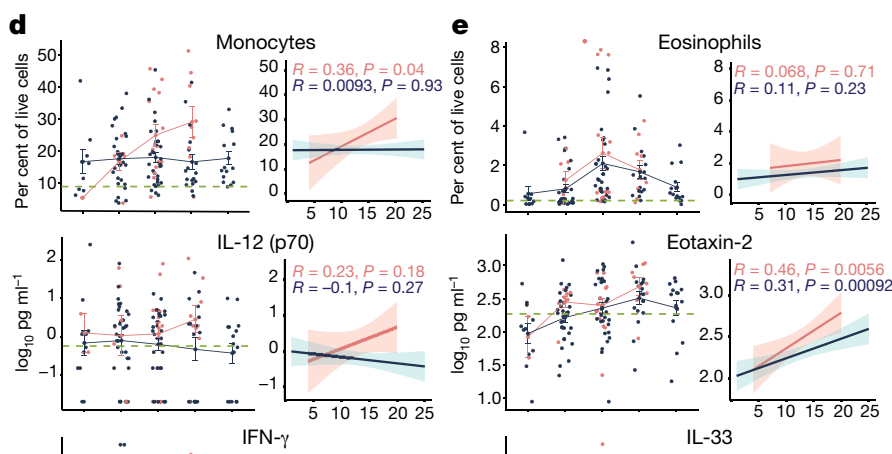

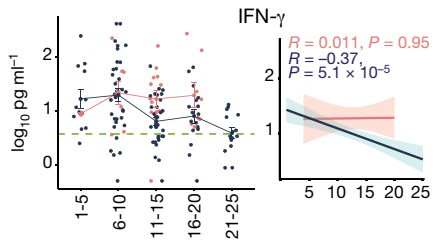

Days from symptom onset
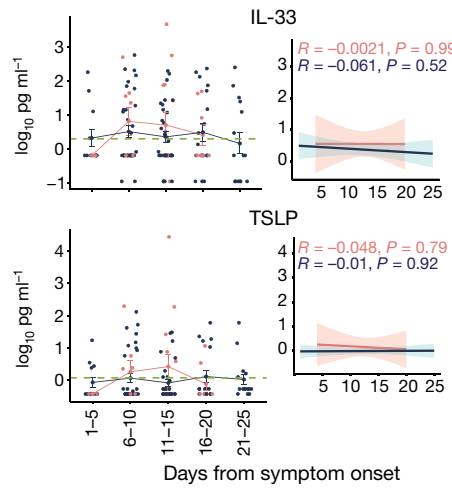

Fig. 2 | Longitudinal immune profiling of moderate and severe COVID-19 patients. a, Correlation matrices of 71 cytokines from patient blood comparing cytokine concentrations in patients with moderate or severe disease during the early phase $(<10 \mathrm{DfSO})$ or late phase $(>10 \mathrm{DfSO})$ of disease. Only significant correlations $(<0.05)$ are represented as dots, and Pearson's correlation coefficient from comparisons of cytokine measurements within each patient is visualized by colour intensity. b, c, Anti-viral interferons (b) and inflammasome-related cytokines (c) plotted as $\log _{10}$ concentrations over time and grouped by disease severity. $\mathbf{d}-\mathbf{f}$, Cellular and cytokine measurements representative of type 1 (d), type 2 (e) and type 3 (f) immune responses

IL-6, which is linked to CRS, was elevated in patients with severe disease $\mathrm{e}^{19}$. Circulating neutrophils did not show a significant increase in our longitudinal analysis (Fig. 2f), although patients with severe disease showed hallmarks of type 3 responses, including increased
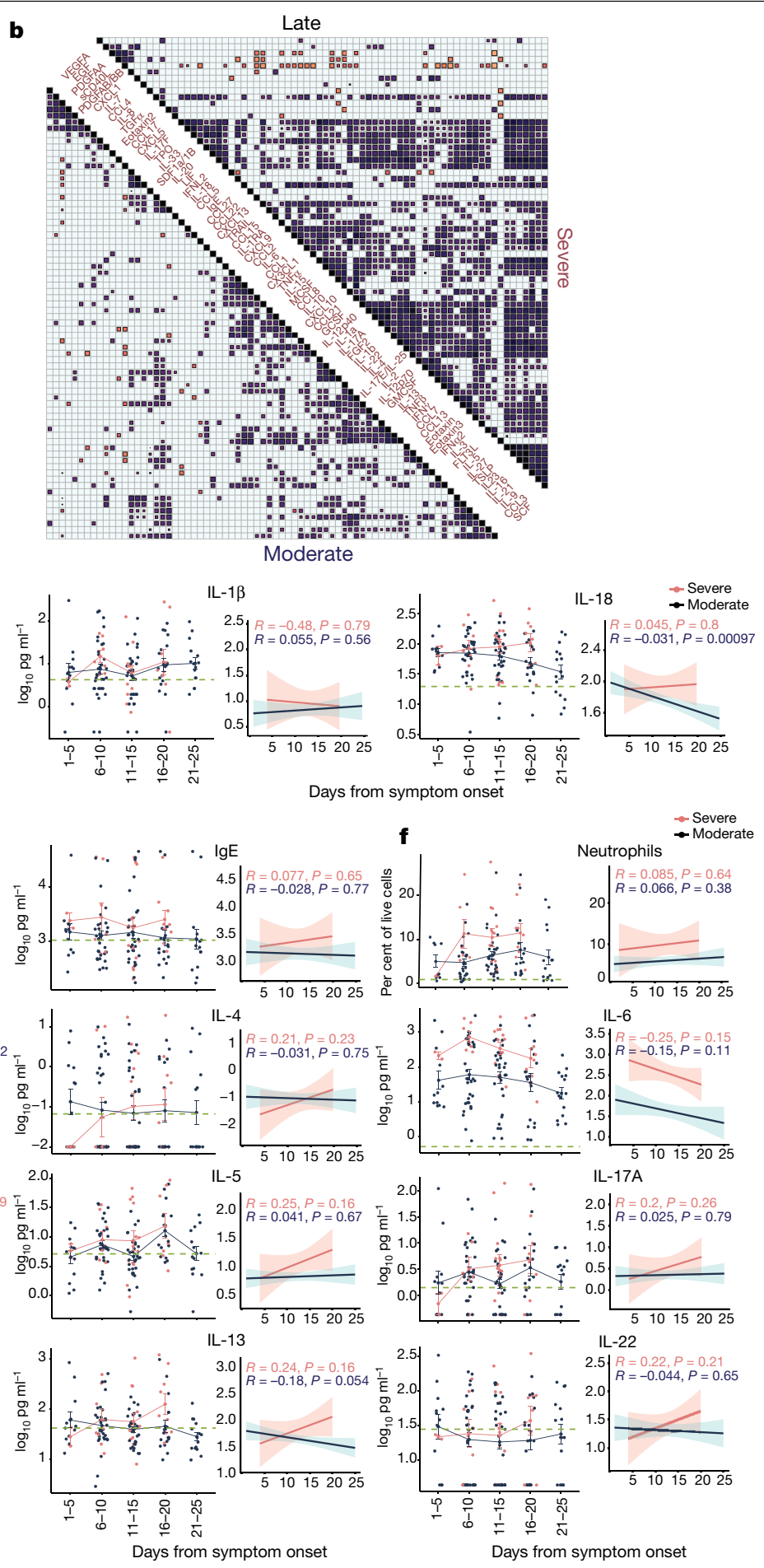

reported over time in intervals of days (left) and continuously as linear regressions (right). Left, each dot represents a distinct patient and time point arranged in intervals of 5 days until 25 DfSO; dark blue, moderate disease $(n=112)$, pink, severe disease $(n=40)$. Dark blue or pink lines pass through the mean at each time interval; error bars denote the s.e.m. Dashed green line, mean from healthy HCWs. Right, regression lines are indicated by the dark blue (moderate) or red (severe) solid lines. Associated Pearson's correlation coefficients and linear regression significance are coloured accordingly; shading represents $95 \% \mathrm{Cl}$.

plasma IL-17A and IL-22, as well as secretion of IL-17 by circulating CD4 T cells as assessed by intracellular cytokine staining (Fig. 2f, Extended Data Fig. 5). These data identify broad elevations of type 1, type 2 and type 3 signatures in severe cases of COVID-19, with differences in their 

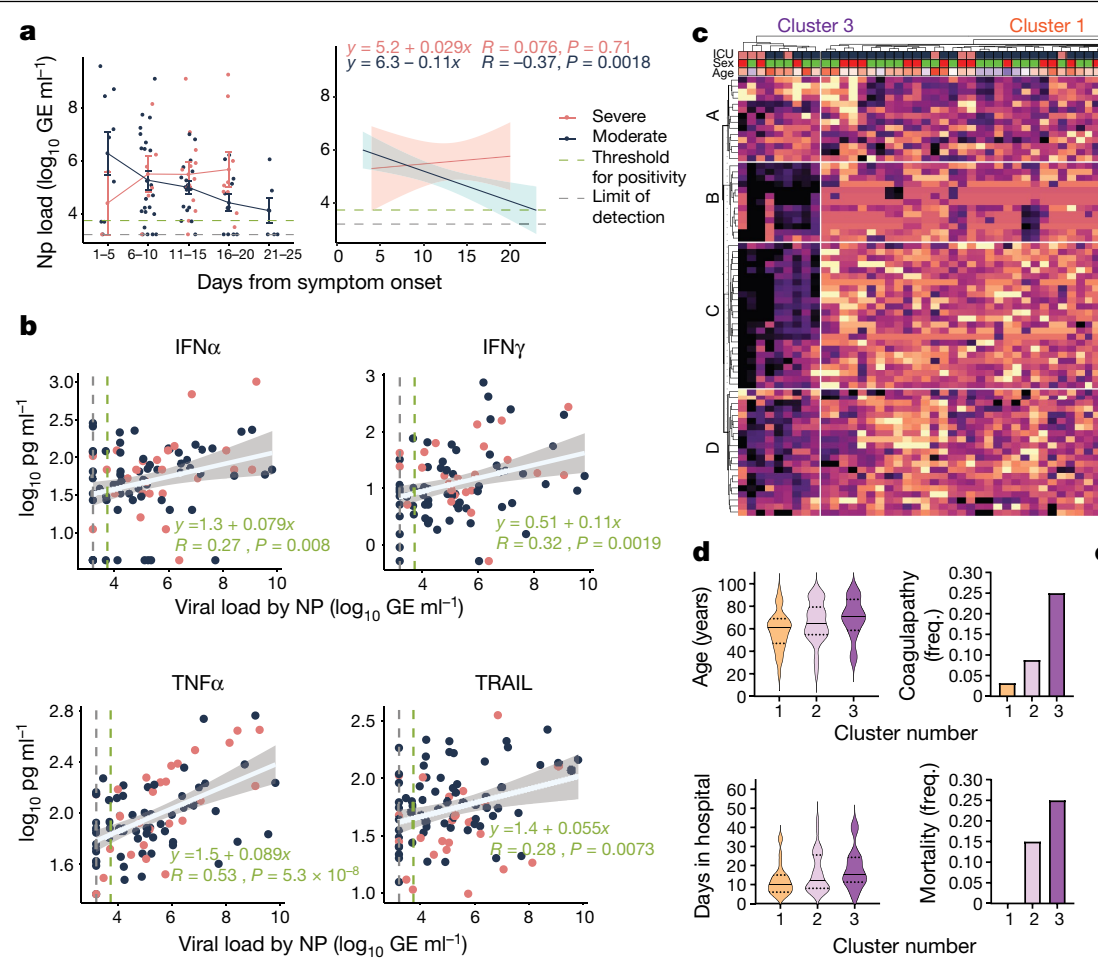

Fig. 3 | Early viral and cytokine profiles distinguish between moderate and severe disease outcomes. a, Viral loads measured by nasopharyngeal swabs are plotted as $\log _{10}$ of genome equivalents against time after symptom onset for patients with moderate disease $(n=112)$ or severe disease $(n=39)$. Left, each dot represents a distinct patient and time point arranged in intervals of 5 days until 25 DfSO. Dark blue or pink lines pass through the mean of each measurement; error bars denote s.e.m. Right, longitudinal data plotted over time continuously. Regression lines are shown as dark blue (moderate) or red (severe). Associated linear regression equations, Pearson's correlation coefficients, and significance are coloured accordingly. Green text is the regression analysis and correlation for all patients. Shading represents $95 \%$ CIs. Dashed green line denotes mean threshold for positivity. Dashed grey line indicates mean limit of detection. b, Correlation and linear regression of cytokines ( $\log _{10}$ concentration) and viral load (by nasopharyngeal swab, $\log _{10}$

genome equivalents (GE)), regardless of disease severity ( $n=151)$. Each dot represents a unique patient time point; dark blue, moderate disease; red, severe disease. White line indicates the regression line for all patients. The associated linear regression equation, Pearson's correlation coefficient, and significance are shown in green. Grey shading indicates $95 \%$ CIs. Dashed green line denotes mean threshold for positivity. Dashed grey line indicates mean limit of detection. c, Unbiased heat map comparisons of cytokines in PBMCs. Measurements were normalized across all patients. $K$-means clustering was used to determine clusters 1-3 (cluster $1, n=46$; cluster $2, n=50$; cluster 3 , $n=16$ ). d, Distribution of age and length of hospital stay (violin plots; solid lines, median; dotted lines, quartiles.) and frequency of coagulopathy and mortality (bar graphs) within each cluster.e, Top 20 cytokines by mutual information analysis to determine their importance for determining mortality. Significance of comparisons determined by two-sided, Wilcoxon rank-sum test.

kinetics and magnitudes between patients with severe and moderate disease.

\section{Viral load correlates with elevated cytokines}

We next measured viral load kinetics using serial nasopharyngeal swabs. Although there was no significant difference in viral RNA load between patients with moderate and severe disease at any specific time point analysed, patients with moderate disease showed a steady decline in viral load over the course of disease, whereas those with severe disease did not (Fig. 3a). Regardless of whether patients exhibited moderate or severe disease, viral load correlated significantly with the levels of IFN $\alpha$, IFN $\gamma$, TNF and tumour necrosis factor-related apoptosis-inducing ligand (TRAIL) (Fig.3b). In addition, several chemokines responsible for monocyte recruitment correlated significantly with viral load only in patients with severe disease (Extended Data Fig. 6a, b). These data indicate that nasopharyngeal viral load correlates with plasma levels of interferons and cytokines.

\section{Early cytokine profile marks disease outcomes}

Next, we investigated whether specific early cytokine responses are associated with severe COVID-19. To this end, we conducted an unsupervised clustering analysis using baseline measurements collected before $12 \mathrm{DfSO}$ (Fig.3c). Three main clusters with correlation to distinct disease outcomes

emerged. These were characterized by four distinct immune signatures. Signature A contained several stromal growth factors, including epidermal growth factor (EGF), platelet-derived growth factor (PDGF) and vascular endothelial growth factor (VEGF), that are mediators of wound healing and tissue repair ${ }^{20}$, as well as IL-7, a key growth factor for lymphocytes. Signature B consisted of eotaxin 3, IL-33 and TSLP, along with IL-21, IL-23 and IL-17F, thus representing type 2 and type 3 immune effectors. Signature C comprised a mixture of all immunotypes, including type 1 (IFN $\gamma$, IL-12 p70, IL-15, IL-2 and TNF), type 2 (IL-4, IL-5 and IL-13), and type 3 cytokines (IL-1 $\alpha$, IL-1ß, IL-17A, IL-17E and IL-22). Finally, signature D contained a number of chemokines involved in leukocyte trafficking, including CCL1, CCL2, CCL5, CCL8, CCL15, CCL21, CCL22, CCL27, CXCL9, CXCL10, CXCL13, and SDF1.

Cluster 1 primarily comprised patients with moderate disease who experienced low occurrences of coagulopathy, shorter lengths of hospital stay, and no mortality (Fig. 3c, d). The main characteristics in this cluster were low levels of inflammatory markers and similar or increased levels of parameters in signature $\mathrm{A}$, which contains tissue reparative growth factors (Fig. 3c). Clusters 2 and 3 were characterized by a rise in inflammatory markers, and patients belonging to these clusters had a higher incidences of coagulopathy and mortality, which was more pronounced in cluster 3 (Fig. 3c, d). Patients in cluster 2 showed higher levels of markers in signatures $C$ and $D$, which included IFN $\alpha$, IL-1Ra and several hallmark type 1 , type 2 and type 3 cytokines, than patients in cluster 1 , but lower expression of markers in signatures $B, C$ and $D$ than those in 


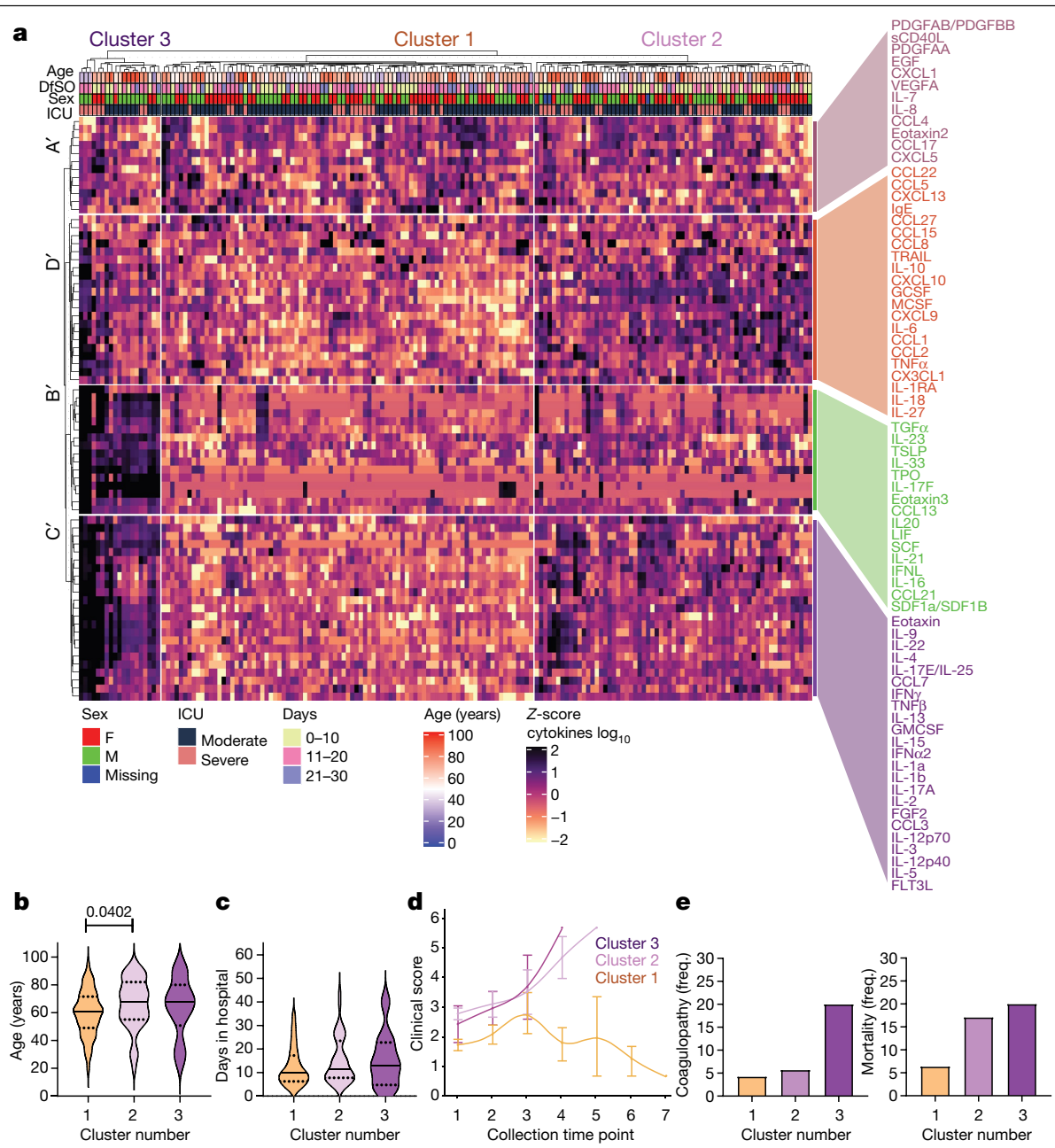

Fig. 4 | Immune correlates of COVID-19 outcomes. a, Unbiased heat map comparisons of cytokines in PBMCs measured at distinct time points in patients with COVID-19. Measurements were normalized across all patients. $K$-means clustering was used to determine clusters 1-3 (cluster $1, n=84$; cluster $2, n=66$; cluster $3, n=20) . \mathbf{b}, \mathbf{c}$, Distribution of age (b) and length of hospital stay (violin plots) (c) of patients within each cluster. For statistical differences, adjusted $P$ values calculated using one-way ANOVA with Tukey's correction for multiple comparisons are shown (age: $F_{(2,90)}=3.115 ; P=0.0492$ ). Solid lines, median; dotted lines, quartiles. d, Disease progression measured by clinical severity score for patients in each cluster. Data (mean \pm s.e.m.) are ordered by the collection time points for each patient, with regular collection intervals of 3-4 days (Extended Data Fig. 7).e, Percentage of patients in each cluster with new-onset coagulopathy or death. cluster 3 (Fig. 3c, d). Patients in cluster 3 showed higher expression of markers in signatures $\mathrm{B}, \mathrm{C}$ and $\mathrm{D}$ than those in other clusters. Cluster 3 showed particular enrichment in expression of markers in signature $B$, including several innate cytokines such as IFN $\lambda$, TGF $\alpha$, TSLP, IL-16, IL-23 and IL-33, and markers linked to coagulopathy, such as TPO (Fig. 3c, d).

We next ranked these parameters obtained at early time points as predictors of severe disease outcomes (Fig. 3e, Extended Data Fig. 6c). In both cases, plasma inflammatory markers were strongly associated with severe disease outcomes. For example, high levels of type IIFN (IFN $\alpha$ ) before the first 12 DfSO correlated with longer hospital stays and death (Fig. 3e, Extended Data Fig. 6c). Moreover, patients who ultimately died of COVID-19 exhibited significantly elevated levels of IFN $\alpha$, IFN $\lambda$ and IL-1Ra, as well as chemokines associated with monocytes and T cell recruitment and survival such as CCL1, CLL2, macrophage colony stimulating factor (M-CSF), IL-2, IL-16 and CCL21, within the first 12 DfSO (Fig. 3e, Extended Data Fig. 6c). These analyses identify specific immunological markers that appear early in the disease and correlate strongly with poor outcomes and death.

\section{Retrospective analysis of immune correlates}

To further evaluate potential drivers of severe COVID-19 outcome in an unbiased manner, we performed unsupervised clustering analysis that included all patients and all time points using cytokines and chemokines (Fig. 4a). Notably, three main clusters of patients emerged and the distribution of patients in early time-point clusters identified in Fig. 3c matched the distribution for the all-time point analysis (Fig. 4a) in $96 \%$ of cases. Cluster 1 primarily comprised patients with moderate disease who showed improving clinical signs (Fig. 4a-d, Extended Data Fig. 7). This cluster contained only two deceased patients. Cluster 1 was characterized by low levels of inflammatory markers as well as similar or increased expression of markers in signature $\mathrm{A}^{\prime}$ (Fig. $4 \mathrm{a}-\mathrm{d}$ ), which mostly matched the signature A markers described in Fig. 3c. Clusters 2 and 3 contained patients with coagulopathy and worsened clinical progression, including most of the deceased patients (Fig. 4a-d, Extended Data Fig. 7).

Clusters 2 and 3 were driven by a set of inflammatory markers that fell into signatures $\mathrm{B}^{\prime}, \mathrm{C}^{\prime}$ and $\mathrm{D}^{\prime}$ to some extent, which overlapped highly with the 'core signature' cytokines and chemokines identified in Fig. 1 as well as with signatures B and C identified in Fig. 3c. These include type 1 immunity markers, including IL-12, chemokines linked to monocyte recruitment and IFNץ; type 2 responses, such as TSLP, chemokines linked to eosinophil recruitment, IL-4, IL-5 and IL-13; and type-3 responses, including IL-23, IL-17A and IL-22. In addition, most CRS- and inflammasome-associated cytokines were enriched in these clusters, including IL-1 $\alpha$, IL-1 $\beta$, IL-6, IL-18 and TNF (Fig. 4a). These 
findings were consistent with generalized estimating equations that identified relationships between the risk of death and cytokines or immune cell populations over time (Extended Data Fig. 8). Together, these results identify groups of inflammatory and potentially protective markers that correlated with COVID-19 trajectories. The immune signatures that correlate with recovery (cluster 1) and the immune signatures that correlate with worsening diseases (cluster $2<$ cluster 3) were remarkably similar whether we took a prospective (Fig. 3) or retrospective (Fig. 4) approach.

\section{Discussion}

Our longitudinal analyses of patients admitted to YNHH with COVID-19 revealed key temporal features of viral load and immune responses that distinguish disease trajectories during hospitalization. Unsupervised clustering revealed three distinct profiles that influenced the evolution and severity of COVID-19. Cluster 1 , characterized by low expression of proinflammatory cytokines and enrichment in tissue repair genes, followed a disease trajectory that remained moderate and led to eventual recovery. Clusters 2 and 3 were characterized by highly elevated proinflammatory cytokines (cluster 3 being more intense), worse disease, and death. Thus, in addition to the known CRS-related pro-inflammatory cytokines, we propose these four signatures of immune response profiles that more accurately divide patients into distinct COVID-19 disease courses.

Although nasopharyngeal viral RNA levels were not significantly different between patients with moderate and severe disease at the specific time points, linear regression analyses showed a slower decline of viral loads in patients who were admitted to the ICU. Viral load was highly correlated with IFN $\alpha$, IFN $\gamma$ and TNF, suggesting that viral load may drive these cytokines and that interferons may not successfully control the viral replication. Moreover, many interferons, cytokines, and chemokines were elevated early in disease for patients who ultimately died of COVID-19. This finding suggests possible pathological roles associated with these host defence factors, as previous reported for patients infected with SARS-CoV- $\mathbf{1}^{21}$.

Our comprehensive analysis of soluble plasma factors revealed broad misfiring of immune effectors in patients with COVID-19, with early predictive markers and distinct dynamics between types of immune responses among moderate and severe disease outcomes. These results suggest that late-stage pathology in COVID-19 may be driven primarily by host immune responses to SARS-CoV-2 and highlights the need for combination therapy to block other cytokines highly represented by these clusters, including inflammasome-dependent cytokines and type 2 cytokines. We observed a correlation with cytokines linked to the inflammasome pathway, which partially overlap with CRS, including IL-1 $\beta$ and IL-18. Indeed, it is plausible that inflammasome activation, along with a sepsis-like CRS, triggers the vascular insults and tissue pathology that are observed in patients with severe COVID-1922.

Overall, our analyses provide a comprehensive examination of the diverse inflammatory dynamics during COVID-19 and possible contributions of distinct sets of inflammatory mediators to disease progression. This raises the possibility that early immunological interventions that target inflammatory markers that are predictive of worse disease outcome would be more beneficial than those that block late-appearing cytokines. Our disease trajectory analyses provide bases for more targeted treatment of patients with COVID-19 based on early cytokine markers, as well as therapies designed to enhance tissue repair and promote disease tolerance.

\section{Online content}

Any methods, additional references, Nature Research reporting summaries, source data, extended data, supplementary information, acknowledgements, peer review information; details of author contributions and competing interests; and statements of data and code availability are available at https://doi.org/10.1038/s41586-020-2588-y.

1. Giamarellos-Bourboulis, E. J. et al. Complex immune dysregulation in COVID-19 patients with severe respiratory failure. Cell Host Microbe 27, 992-1000.e3 (2020).

2. Zhou, Z. et al. heightened innate immune responses in the respiratory tract of COVID-19 patients. Cell Host Microbe 27, 883-890.e2 (2020).

3. Huang, C. et al. Clinical features of patients infected with 2019 novel coronavirus in Wuhan, China. Lancet 395, 497-506 (2020).

4. Mathew, D. et al. Deep immune profiling of COVID-19 patients reveals distinct immunotypes with therapeutic implications. Science https://doi.org/10.1126/science. abc8511 (2020).

5. Hoffmann, M. et al. SARS-CoV-2 cell entry depends on ACE2 and TMPRSS2 and is blocked by a clinically proven protease inhibitor. Cell 181, 271-280.e8 (2020).

6. Yan, R. et al. Structural basis for the recognition of SARS-CoV-2 by full-length human ACE2. Science 367, 1444-1448 (2020).

7. Chen, J. \& Subbarao, K. The Immunobiology of SARS. Annu. Rev. Immunol. 25, 443-472 (2007).

8. Jose, R. J. \& Manuel, A. COVID-19 cytokine storm: the interplay between inflammation and coagulation. Lancet Respir. Med. 8, e46-e47 (2020).

9. Chen, G. et al. Clinical and immunological features of severe and moderate coronavirus disease 2019. J. Clin. Invest. 130, 2620-2629 (2020).

10. Chen, N. et al. Epidemiological and clinical characteristics of 99 cases of 2019 novel coronavirus pneumonia in Wuhan, China: a descriptive study. Lancet 395, 507-513 (2020).

11. Annunziato, F., Romagnani, C. \& Romagnani, S. The 3 major types of innate and adaptive cell-mediated effector immunity. J. Allergy Clin. Immunol. 135, 626-635 (2015).

12. Iwasaki, A. \& Medzhitov, R. Control of adaptive immunity by the innate immune system. Nat. Immunol. 16, 343-353 (2015).

13. O'Shea, J. J. \& Paul, W. E. Mechanisms underlying lineage commitment and plasticity of helper CD4+ T cells. Science 327, 1098-1102 (2010).

14. Ostendorf, L. et al. Low-density granulocytes are a novel immunopathological feature in both multiple sclerosis and neuromyelitis optica spectrum disorder. Front. Immunol. 10, 2725 (2019).

15. Kalfaoglu, B., Almeida-Santos, J., Adele Tye, C., Satou, Y. \& Ono, M. T-cell hyperactivation and paralysis in severe COVID-19 infection revealed by single-cell analysis. Preprint at https://www.biorxiv.org/content/10.1101/2020.05.26.115923v2 (2020).

16. Blanco-Melo, D. et al. Imbalanced host response to SARS-CoV-2 drives development of COVID-19. Cell 181, 1036-1045.e1039 (2020).

17. Kuri-Cervantes, L. et al. Comprehensive mapping of immune perturbations associated with severe COVID-19. Sci. Immunol. 15, eabd7114 (2020).

18. Gabay, C., Lamacchia, C. \& Palmer, G. IL-1 pathways in inflammation and human diseases. Nat. Rev. Rheumatol. 6, 232-241 (2010).

19. Wang, D. et al. The regulation of the Treg/Th17 balance by mesenchymal stem cells in human systemic lupus erythematosus. Cell. Mol. Immunol. 14, 423-431 (2017).

20. Duffield, J. S., Lupher, M., Thannickal, V. J. \& Wynn, T. A. Host responses in tissue repair and fibrosis. Annu. Rev. Pathol. 8, 241-276 (2013).

21. Cameron, M. J. et al. Interferon-mediated immunopathological events are associated with atypical innate and adaptive immune responses in patients with severe acute respiratory syndrome. J. Virol. 81, 8692-8706 (2007).

22. Yap, J. K. Y., Moriyama, M. \& Iwasaki, A. Inflammasomes and pyroptosis as therapeutic targets for COVID-19. J. Immunol. 205, 307-312 (2020).

Publisher's note Springer Nature remains neutral with regard to jurisdictional claims in published maps and institutional affiliations.

(c) The Author(s), under exclusive licence to Springer Nature Limited 2020

Yale IMPACT Research Team

Abeer Obaid" ${ }^{11}$ Alice Lu-Culligan', Allison Nelson"1, Anderson Brito ${ }^{3}$, Angela Nunez" Anjelica Martin', Annie Watkins ${ }^{3}$, Bertie Geng ${ }^{11}$, Chaney Kalinich ${ }^{3}$, Christina Harden ${ }^{3}$, Codruta Todeasa", Cole Jensen ${ }^{3}$, Daniel Kim", David McDonald", Denise Shepard", Edward Courchaine $^{12}$, Elizabeth B. White ${ }^{3}$, Eric Song', Erin Silva" , Eriko Kudo', Giuseppe Deluliis', Harold Rahming", Hong-Jai Park", Irene Matos", Jessica Nouws", Jordan Valdez"1, Joseph Fauver $^{3}$, Joseph Lim ${ }^{13}$, Kadi-Ann Rose" ${ }^{11}$, Kelly Anastasio ${ }^{14}$, Kristina Brower ${ }^{3}$, Laura Glick ${ }^{11}$, Lokesh Sharma", Lorenzo Sewanan" , Lynda Knaggs", Maksym Minasyan", Maria Batsu", Mary Petrone ${ }^{3}$, Maxine Kuang ${ }^{3}$, Maura Nakahata", Melissa Campbell ${ }^{8}$, Melissa Linehan', Michael H. Askenase ${ }^{15}$, Michael Simonov" ${ }^{11}$, Mikhail Smolgovsky ${ }^{11}$, Nicole Sonnert', Nida Naushad", Pavithra Vijayakumar", Rick Martinello", Rupak Datta ${ }^{4}$, Ryan Handoko", Santos Bermejo", Sarah Prophet ${ }^{16}$, Sean Bickerton ${ }^{12}$, Sofia Velazquez ${ }^{15}$, Tara Alpert ${ }^{4}$, Tyler Rice', William Khoury-Hanold', Xiaohua Peng", Yexin Yang', Yiyun Cao' \& Yvette Strong"

${ }^{11}$ Yale University School of Medicine, New Haven, CT, USA. ${ }^{12}$ Department of Biochemistry and of Molecular Biology, Yale University School of Medicine, New Haven, CT, USA. ${ }^{13}$ Yale Viral Hepatitis Program, Yale University School of Medicine, New Haven, CT, USA. ${ }^{14}$ Yale Center for Clinical Investigation, Yale University School of Medicine, New Haven, CT, USA. ${ }^{15}$ Department of Neurology, Yale University School of Medicine, New Haven, CT, USA. ${ }^{16}$ Department of Molecular, Cellular and Developmental Biology, Yale University School of Medicine, New Haven, CT, USA. 


\section{Methods}

\section{Ethics statement}

This study was approved by Yale Human Research Protection Program Institutional Review Boards (FWA00002571, protocol ID 2000027690). Informed consent was obtained from all enrolled patients and healthcare workers.

\section{Patients}

One-hundred and thirty-five patients admitted to YNHH with COVID19 between 18 March 2020 and 5 May 2020 were included in this study. No statistical methods were used to predetermine sample size. Nasopharyngeal swabs were collected as described ${ }^{23}$, approximately every four days, for SARS-CoV-2 RT-qPCR analysis where clinically feasible. Paired whole blood for flow cytometry analysis was collected simultaneously in sodium heparin-coated vacutainers and kept on gentle agitation until processing. All blood was processed on the day of collection. Patients were scored for COVID-19 disease severity through review of electronic medical records (EMR) at each longitudinal time point. Scores were assigned by a clinical infectious disease physician according to a custom-developed disease severity scale. Moderate disease status (clinical score 1-3) was defined as:SARS-CoV-2 infection requiring hospitalization without supplementary oxygen (1); infection requiring non-invasive supplementary oxygen $(<3 \mathrm{l} / \mathrm{min}$ to maintain $\mathrm{SpO}_{2}>92 \%$ ) (2); and infection requiring non-invasive supplementary oxygen ( $>3 \mathrm{l} / \mathrm{min}$ to maintain $\mathrm{SpO}_{2}>92 \%$, or $>2 \mathrm{l} / \mathrm{min}$ to maintain $\mathrm{SpO}_{2}>92 \%$ and had a high-sensitivity C-reactive protein $(\mathrm{CRP})>70$ ) and received tocilizumab). Severe disease status (clinical score 4 or 5) was defined as infection meeting all criteria for clinical score 3 and also requiring admission to the ICU and $>6 \mathrm{l} / \mathrm{min}$ supplementary oxygen to maintain $\mathrm{SpO}_{2}>92 \%$ (4); or infection requiring invasive mechanical ventilation or extracorporeal membrane oxygenation (ECMO) in addition to glucocorticoid or vasopressor administration (5). Clinical score 6 was assigned for deceased patients. Of note, the use of tocilizumab can increase circulating levels of IL- 6 by inhibiting IL-6R $\alpha$-mediated degradation. Analysis of our cohort indicate higher plasma levels of IL-6 in patients with either moderate or severe disease who received tocilizumab treatment (Extended Data Fig. 1d).

For all patients, days from symptom onset were estimated as follows: (1) highest priority was given to explicit onset dates provided by patients; (2) next highest priority was given to the earliest reported symptom by a patient; and (3) in the absence of direct information regarding symptom onset, we estimated a date through manual assessment of the electronic medical record (EMRs) by an independent clinician. Demographic information was aggregated through a systematic and retrospective review of patient EMRs and was used to construct Extended Data Table 1. Symptom onset and aetiology were recorded through standardized interviews with patients or patient surrogates upon enrollment in our study, or alternatively through manual EMR review if no interview was possible owing to clinical status. The clinical data were collected using EPIC EHR and REDCap 9.3.6 software. At the time of sample acquisition and processing, investigators were unaware of the patients' conditions. Blood acquisition was performed and recorded by a separate team. Information about patients' conditions was not available until after processing and analysis of raw data by flow cytometry and ELISA. A clinical team, separate from the experimental team, performed chart reviews to determine relevant statistics. Cytokines and FACS analyses were performed blinded. Patients' clinical information and clinical score coding were revealed only after data collection.

\section{Viral RNA measurements}

RNA concentrations were measured from nasopharyngeal samples by RT-qPCR as previously described ${ }^{23}$. In brief, total nucleic acid was extracted from $300 \mu \mathrm{l}$ of viral transport medium (nasopharyngeal swab) using the MagMAX Viral/Pathogen Nucleic Acid Isolation kit (ThermoFisher Scientific) with a modified protocol and eluted into $75 \mu$ l elution buffer.

To detect SARS-CoV-2 RNA, we tested $5 \mu$ RNA 371 template as previously described ${ }^{24}$, using the US CDC real-time RT-qPCR primer/probe sets for 2019-nCoV_N1, 2019-nCoV_N2, and the human RNase P(RP) as an extraction control. Virus RNA copies were quantified using a tenfold dilution standard curve of RNA transcripts that we previously generated $^{24}$. The lower limit of detection for SARS-CoV-2 genomes assayed by qPCR in nasopharyngeal specimens was established as described ${ }^{24}$. In addition to a technical detection threshold, we also used a clinical referral threshold (detection limit) to either: (1) refer asymptomatic HCWs for diagnostic testing at a CLIA-approved laboratory; or (2) cross-validate results from a CLIA-approved laboratory for SARS-CoV-2 qPCR-positive individuals upon study enrollment. Individuals above the technical detection threshold, but below the clinical referral threshold, were considered SARS-CoV-2 positive for the purposes of our research.

\section{Isolation of patient plasma}

Plasma samples were collected after centrifugation of whole blood at $400 \mathrm{~g}$ for $10 \mathrm{~min}$ at room temperature (RT) without brake. The undiluted serum was then transferred to $15-\mathrm{ml}$ polypropylene conical tubes, and aliquoted and stored at $-80^{\circ} \mathrm{C}$ for subsequent analysis.

\section{Cytokine and chemokine measurements}

Patient serum was isolated as before and aliquots were stored at $-80^{\circ} \mathrm{C}$. Sera were shipped to Eve Technologies (Calgary, Alberta, Canada) on dry ice, and levels of cytokines and chemokines were measured using the Human Cytokine Array/Chemokine Array 71-403 Plex Panel (HD71). All samples were measured upon the first thaw.

\section{Isolation of PBMCs}

PBMCs were isolated from heparinized whole blood using Histopaque (Sigma-Aldrich, \#10771-500ML) density gradient centrifugation in a biosafety level $2+$ facility. After isolation of undiluted serum, blood was diluted 1:1 in room temperature PBS, layered over Histopaque in a SepMate tube (StemCell Technologies; \#85460) and centrifuged for 10 min at $1,200 \mathrm{~g}$. The PBMC layer was isolated according to the manufacturer's instructions. Cells were washed twice with PBS before counting. Pelleted cells were briefly treated with ACK lysis buffer for 2 min and then counted. Percentage viability was estimated using standard Trypan blue staining and an automated cell counter (Thermo-Fisher, \#AMQAX1000).

\section{Flow cytometry}

Antibody clones and vendors were as follows: BB515 anti-hHLA-DR(G466) (1:400) (BD Biosciences), BV785 anti-hCD16 (3G8) (1:100) (BioLegend), PE-Cy7 anti-hCD14 (HCD14) (1:300) (BioLegend), BV605 anti-hCD3 (UCHT1) (1:300) (BioLegend), BV711 anti-hCD19 (SJ25C1) (1:300) (BD Biosciences), AlexaFluor647 anti-hCD1c (L161) (1:150) (BioLegend), biotin anti-hCD141 (M80) (1:150) (BioLegend), PE-Dazzle594 anti-hCD56 (HCD56) (1:300) (BioLegend), PE anti-hCD304 (12C2) (1:300) (BioLegend), APCFire750 anti-hCD11b (ICRF44) (1:100) (BioLegend), PerCP/ Cy5.5 anti-hCD66b (G10F5) (1:200) (BD Biosciences), BV785 anti-hCD4 (SK3) (1:200) (BioLegend), APCFire750 or PE-Cy7 or BV711 anti-hCD8 (SK1) (1:200) (BioLegend), BV421 anti-hCCR7 (G043H7) (1:50) (BioLegend), AlexaFluor 700 anti-hCD45RA (HI100) (1:200) (BD Biosciences), PE anti-hPD1 (EH12.2H7) (1:200) (BioLegend), APC anti-hTIM3 (F38-2E2) (1:50) (BioLegend), BV711 anti-hCD38(HIT2) (1:200) (BioLegend), BB700 anti-hCXCR5 (RF8B2) (1:50) (BD Biosciences), PE-Cy7 anti-hCD127 (HIL-7R-M21) (1:50) (BioLegend), PE-CF594 anti-hCD25 (BC96) (1:200) (BD Biosciences), BV711 anti-hCD127 (HIL-7R-M21) (1:50) (BD Biosciences), BV421 anti-hIL17a (N49-653) (1:100) (BD Biosciences), AlexaFluor 700 anti-hTNFa (MAb11) (1:100) (BioLegend), PE or APC/Fire750 anti-hIFNy (4S.B3) (1:60) (BioLegend), FITC anti-hGranzymeB (GB11) 
(1:200) (BioLegend), AlexaFluor 647 anti-hIL-4 (8D4-8) (1:100) (BioLegend), BB700 anti-hCD183/CXCR3 (1C6/CXCR3) (1:100) (BDBiosciences), PE-Cy7 anti-hIL-6 (MQ2-13A5) (1:50) (BioLegend), PE anti-hIL-2 (5344.111) (1:50) (BD Biosciences), BV785 anti-hCD19 (SJ25C1) (1:300) (BioLegend), BV421 anti-hCD138 (MI15) (1:300) (BioLegend), AlexaFluor700 anti-hCD20 (2H7) (1:200) (BioLegend), AlexaFluor 647 anti-hCD27 (M-T271) (1:350) (BioLegend), PE/Dazzle594 anti-hIgD (IA6-2) (1:400) (BioLegend), PE-Cy7 anti-hCD86 (IT2.2) (1:100) (BioLegend), APC/ Fire750 anti-hIgM (MHM-88) (1:250) (BioLegend), BV605 anti-hCD24 (ML5) (1:200) (BioLegend), BV421 anti-hCD10 (HI1Oa) (1:200) (BioLegend), BV421 anti-CDh15 (SSEA-1) (1:200) (BioLegend), AlexaFluor 700 Streptavidin (1:300) (ThermoFisher), BV605 Streptavidin (1:300) (BioLegend). In brief, freshly isolated PBMCs were plated at $1-2 \times 10^{6}$ cells per well in a 96-well U-bottom plate. Cells were resuspended in Live/Dead Fixable Aqua (ThermoFisher) for $20 \mathrm{~min}$ at $4{ }^{\circ} \mathrm{C}$. Following a wash, cells were blocked with Human TruStan FcX (BioLegend) for $10 \mathrm{~min}$ at RT. Cocktails of desired staining antibodies were added directly to this mixture for $30 \mathrm{~min}$ at RT. For secondary stains, cells were first washed and supernatant aspirated; then to each cell pellet a cocktail of secondary markers was added for $30 \mathrm{~min}$ at $4{ }^{\circ} \mathrm{C}$. Prior to analysis, cells were washed and resuspended in $100 \mu \mathrm{l} 4 \%$ PFA for 30 min at $4{ }^{\circ} \mathrm{C}$. For intracellular cytokine staining following stimulation, cells were resuspended in $200 \mu \mathrm{l}$ cRPMI (RPMI-1640 supplemented with $10 \%$ FBS, 2 mM L-glutamine, $100 \mathrm{U} / \mathrm{ml}$ penicillin, and $100 \mathrm{mg} / \mathrm{ml}$ streptomycin, $1 \mathrm{mM}$ sodium pyruvate, and $50 \mu \mathrm{M} 2$-mercaptoethanol) and stored at $4{ }^{\circ} \mathrm{C}$ overnight. Subsequently, these cells were washed and stimulated with $1 \times$ Cell Stimulation Cocktail (eBioscience) in $200 \mu \mathrm{l}$ cRPMI for $1 \mathrm{~h}$ at $37^{\circ} \mathrm{C}$. Fifty microlitres of $5 \times$ Stimulation Cocktail (plus protein transport 442 inhibitor) (eBioscience) was added for an additional $4 \mathrm{~h}$ of incubation at $37^{\circ} \mathrm{C}$. Following stimulation, cells were washed and resuspended in $100 \mu \mathrm{l} 4 \%$ PFA for $30 \mathrm{~min}$ at $4^{\circ} \mathrm{C}$. To quantify intracellular cytokines, these samples were permeabilized with $1 \times$ permeabilization buffer from the FOXP3/Transcription Factor Staining Buffer Set (eBioscience) for $10 \mathrm{~min}$ at $4{ }^{\circ} \mathrm{C}$. All subsequent staining cocktails were made in this buffer. Permeabilized cells were then washed and resuspended in a cocktail containing Human TruStan FcX (BioLegend) for 10 min at $4{ }^{\circ} \mathrm{C}$. Finally, intracellular staining cocktails were added directly to each sample for $1 \mathrm{~h}$ at $4{ }^{\circ} \mathrm{C}$. Following this incubation, cells were washed and prepared for analysis on an Attune NXT (ThermoFisher). Data were analysed using FlowJo software version 10.6 software (Tree Star). The specific sets of markers used to identify each subset of cells are summarized in Extended Data Fig. 9.

\section{Statistical analysis}

Patients and their analysed features were clustered using the $K$-means algorithm. Heat maps were created using the ComplexHeatmap package $^{25}$. The optimum number of clusters was determined by using the silhouette coefficient analysis, available with the NBClust and factoextra packages ${ }^{26}$. Before data visualization, each feature was scaled and centred. Multiple group comparisons were analysed by running both parametric (ANOVA) and non-parametric (Kruskal-Wallis) statistical tests with Dunn's and Tukey's post hoc tests. Mutual information analyses were performed using the Caret $\mathrm{R}$ package and visualized using ggplot2. Multiple correlation analysis was performed by computing
Spearman's coefficients with the Hmisc package for R and visualized with corrplot by only showing correlations with $P<0.05$. For generalized linear models (GLM), we calculated the incident risk ratio (IRR) by conducting a Poisson regression with a log link and robust variance estimation; this value approximates the risk ratio estimated by a log-linear model. For generalized estimating equation (GEE) models, we calculated the incidence risk ratio (IRR) in the same way as for non-GEE GLM models, assuming an independent correlation structure. All models controlled for participant sex and age.

\section{Reporting summary}

Further information on research design is available in the Nature Research Reporting Summary linked to this paper.

\section{Data availability}

All the background information on HCWs, clinical information for patients, and raw data used in this study are included in Supplementary Table 1. Additionally, all of the raw fcs files for the flow cytometry analysis are available at ImmPort (https://www.immport.org/shared/ home; study ID SDY1655).

23. Wyllie, A. L. et al. Saliva is more sensitive for SARS-CoV-2 detection in COVID-19 patients than nasopharyngeal swabs. Preprint at https://www.medrxiv.org/content/10.1101/2020.0 4.16.20067835v1 (2020).

24. Vogels, C. B. F. et al. Analytical sensitivity and efficiency comparisons of SARS-COV-2 qRT-PCR primer-probe sets. Nat. Microbiol. https://doi.org/10.1038/s41564-020-0761-6 (2020).

25. Gu, Z., Eils, R. \& Schlesner, M. Complex heatmaps reveal patterns and correlations in multidimensional genomic data. Bioinformatics 32, 2847-2849 (2016).

26. Charrad, M., Ghazzali, N., Boiteau, V. \& Niknafs, A. NbClust: An R package for determining the relevant number of clusters in a data set. J. Stat. Softw. 61, 1-36 (2014).

Acknowledgements We thank M. Linehan for technical and logistical assistance, and A. Wang, A. Ring, C. Wilen and D. Mucida for discussions. This work was supported by the Women's Health Research at Yale Pilot Project Program (A.I.), Fast Grant from Emergent Ventures at the Mercatus Center, Mathers Foundation, and the Ludwig Family Foundation, the Department of Internal Medicine at the Yale School of Medicine, Yale School of Public Health and the Beatrice Kleinberg Neuwirth Fund. IMPACT received support from the Yale COVID-19 Research Resource Fund. A.I. is an Investigator of the Howard Hughes Medical Institute. C.L. is a Pew Latin American Fellow. P.W. is supported by Gruber Foundation and the NSF. B.I. is supported by NIAID 2T32AI007517-16. C.B.F.V. is supported by NOW Rubicon 019.181EN.004.

Author contributions A.I.K. and A.I. conceived the study. C.L., P.W., J.K., J.S., J.E.O. S.M., H.W. and T.M. defined parameters, collected and processed patient PBMC samples and analysed data. T.B.R.C. performed bioinformatic analysis. B.I., J.K. T.T. and C.D.O. collected epidemiological and clinical data. A.L.W., C.B.F.V., I.M.O., R.E., S.L., P.L., A.V., A.P. and M.T performed the virus RNA concentration assays. N.D.G. supervised the virus RNA concentration assays. A.C.-M., M.C.M and A.J.M. processed and stored patient specimens. J.B.F., C.D.C. M.C. and S.F. assisted in patient and HCW recruitment. W.L.S. supervised clinical data management. M.S., M.K.E. and S.B.O. carried out statistical analyses. A.C.S. and R.M. contributed personnel, equipment and insights. C.L. and A.I. drafted the manuscript. All authors helped to edit the manuscript. A.I. and R.H. secured funds. A.I. and S.B.O. supervised the project.

Competing interests The authors declare no competing interests.

Additional information

Supplementary information is available for this paper at https://doi.org/10.1038/s41586-0202588-y.

Correspondence and requests for materials should be addressed to $A . I$.

Peer review information Nature thanks Petter Brodin, Malik Peiris and the other, anonymous, reviewer(s) for their contribution to the peer review of this work. Peer reviewer reports are available.

Reprints and permissions information is available at http://www.nature.com/reprints. 


\section{Article}

A

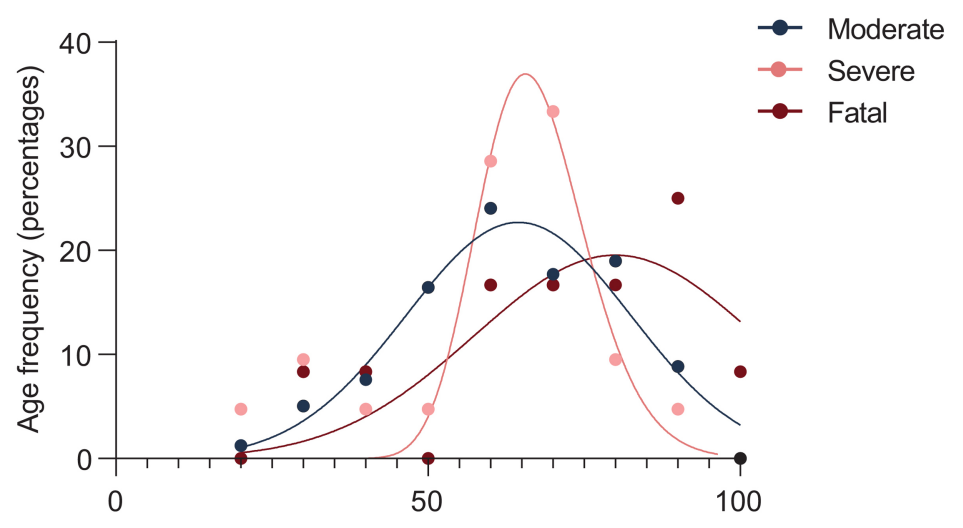

C

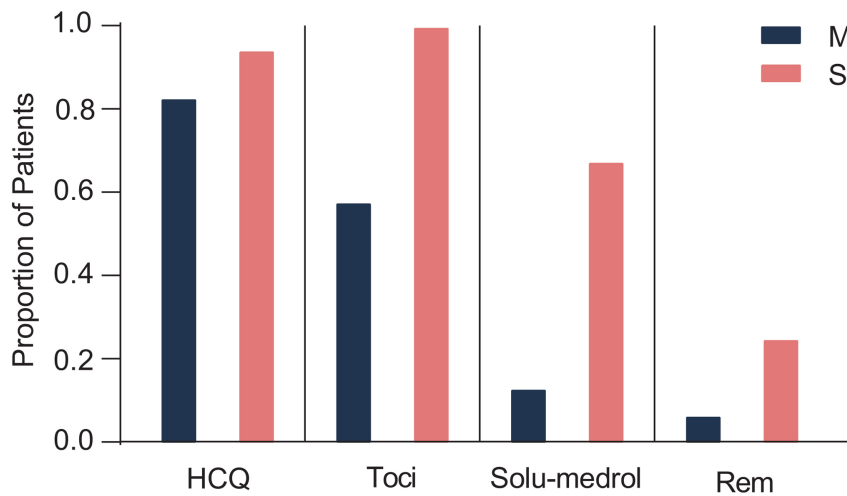

Extended Data Fig. $1 \mid$ Age and BMI cohort distributions and Select Medications distributions. a, b, Aggregated ages (a) and BMIs (b) were collected for patients with moderate, severe, and fatal COVID-19 and relative frequency histograms generated for comparison across disease sub-groups. Gaussian and lognormal distributions were fit through least squares regression and compared for goodness of fit through differential Akaike
B

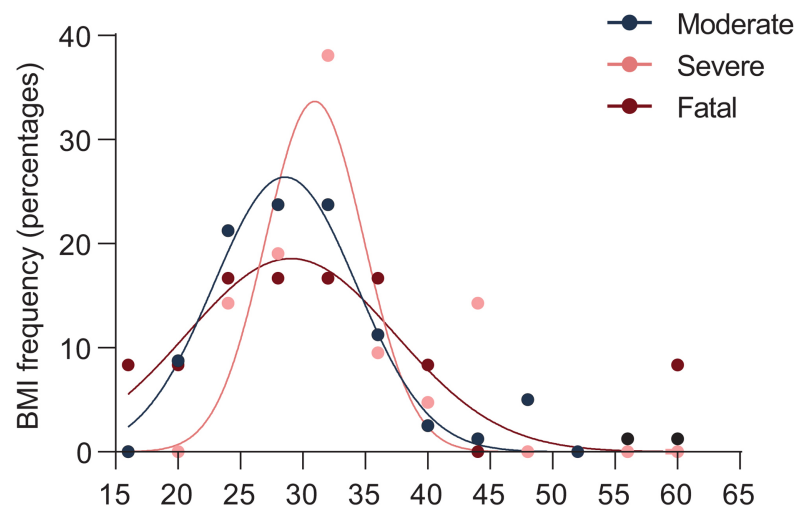

$\mathrm{D}$

Changes in IL-6, according to tocilizumab, after controlling, age, ICU admission and days from symptom onset, accounting for multiple observations per person (GEE).

\begin{tabular}{lll}
\hline Covariate & Coefficient & $95 \% \mathrm{Cl}$ \\
\hline Tocilizumab & $\mathbf{0 . 5 5}$ & $\mathbf{0 . 0 7}, 1.04$ \\
ICU admission & 0.48 & $-0.02,0.99$ \\
Days from symptom onset & -0.02 & $-0.04,-0.003$ \\
Age & 0.01 & $0.0003,0.02$ \\
\hline This analysis excludes two individuals whose IL-6 was measured before receiving tocilizumab.
\end{tabular}

Changes in T cell count, according to age, ICU admission and days from symptom onset, accounting for multiple observations per person (GEE).

\begin{tabular}{lll}
\hline Covariate & Coefficient & $95 \% \mathrm{Cl}$ \\
\hline ICU admission & -13.43 & $-19.32,-7.55$ \\
Days from symptom onset & -0.15 & $-0.41,0.11$ \\
Age & $-\mathbf{0 . 1 6}$ & $\mathbf{- 0 . 3 3 , 0 . 0 2}$ \\
\hline
\end{tabular}

information criterion (AICc) comparison. All distributions were best described by a Gaussian model except for age in the 'severe' disease category, which was best modelled by a lognormal distribution. c, Proportion of patients admitted to YNHH receiving hydroxycholorquine (HCQ), tocilizumab (Toci), methylprednisolone (Solu-medrol), and remdesivir (Rem) are shown, stratified by disease severity. d, Medication and age adjustments for IL- 6 and T cell count. 

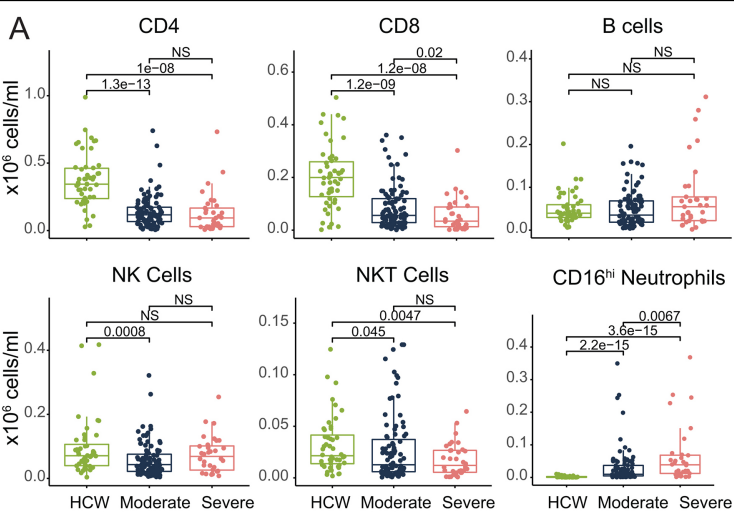

CD16 ${ }^{\text {hi }}$ Neutrophils
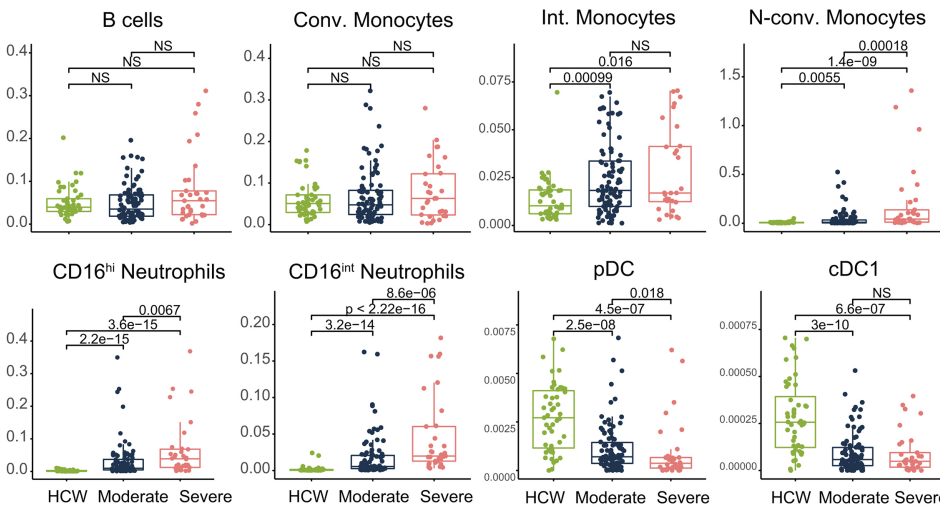

B Naive
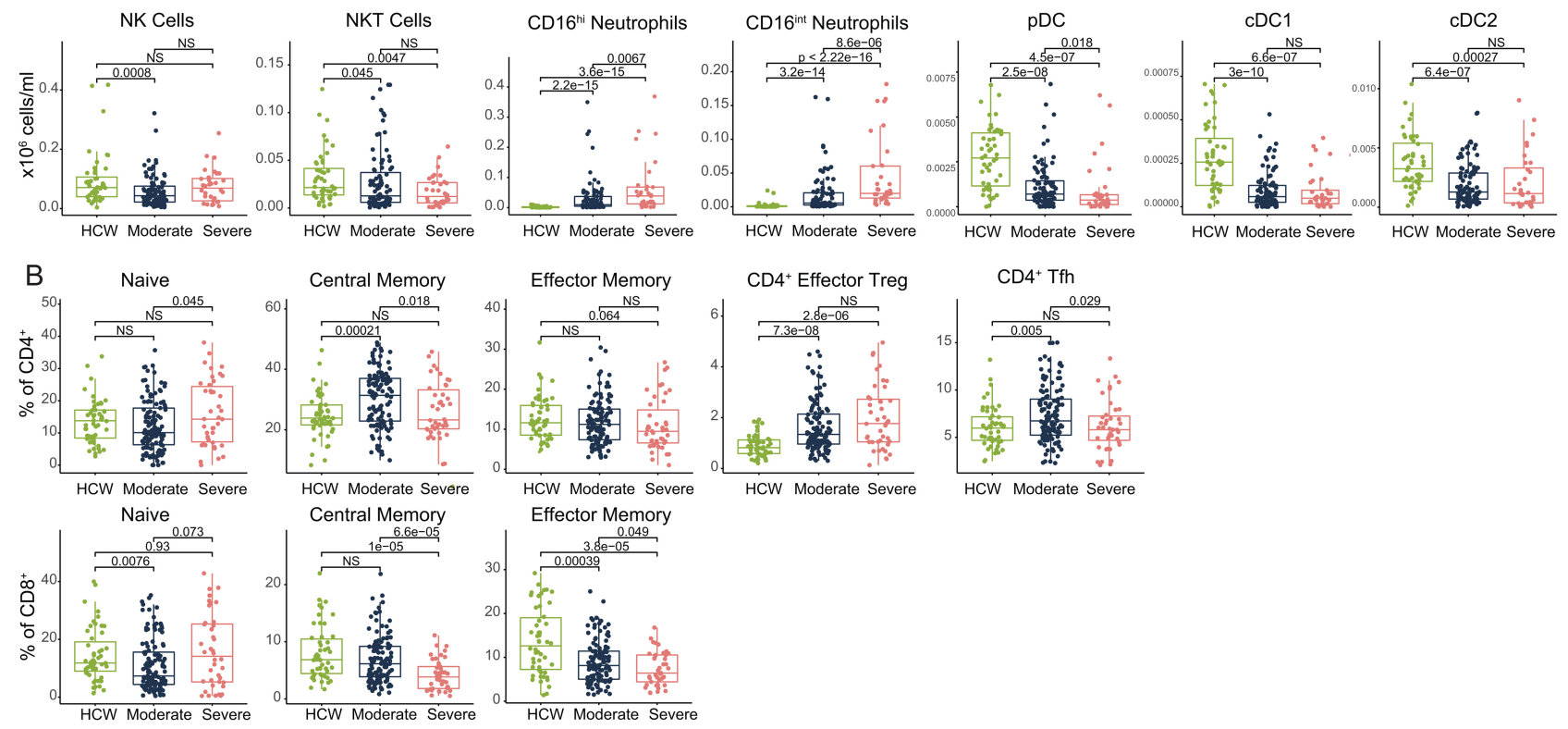

Effector Memory
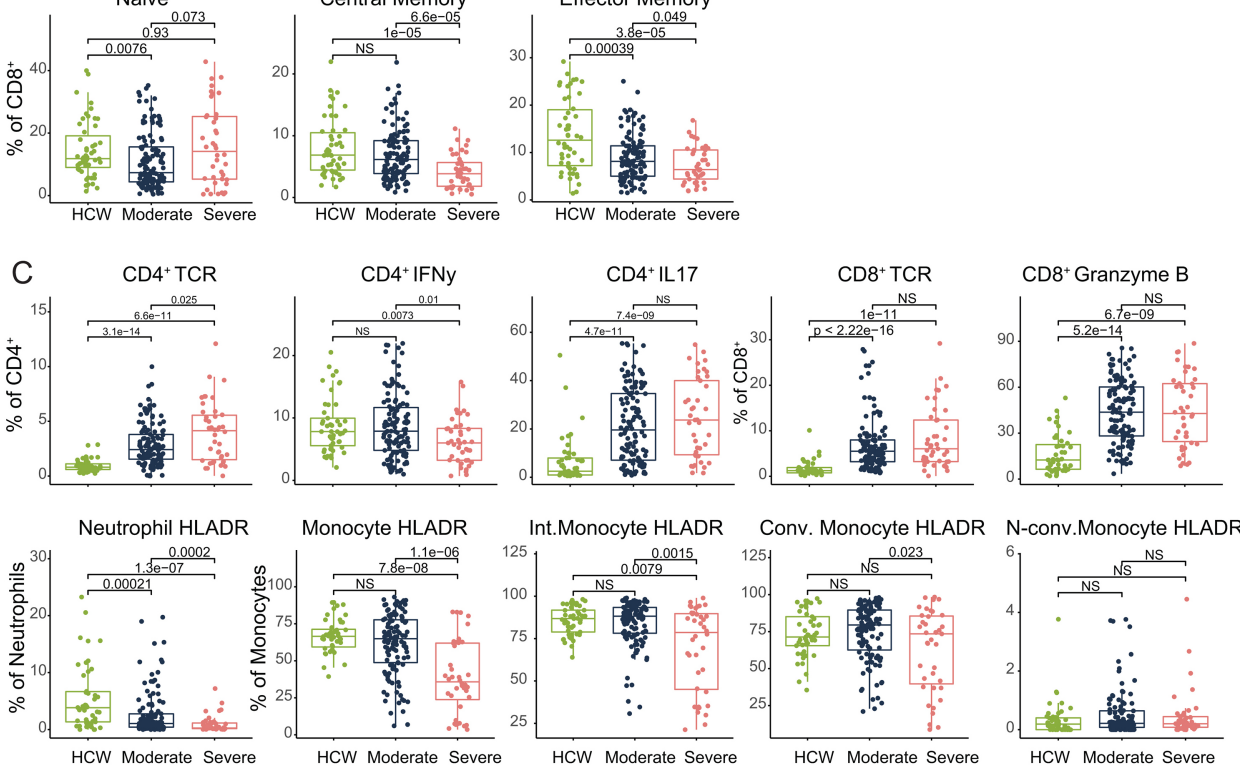

Extended Data Fig. 2 | Overview of cellular immune changes in COVID-19 patients. a, b, Immune cell subsets of interest, plotted as a concentration of millions of cells per millilitre of blood (a) or as a percentage of a parent population (b).c, Phenotyping to TCR-activated T cells, cytokine-secreting T cells, and HLA-DR expression within monocytes and neutrophils. Each dot represents a separate time point per subject (HCW, $n=49$; Moderate, $n=114$;
Severe, $n=41)$. For all boxplots, the centre is drawn through the median of the measurement, and the lower and upper bounds of the box correspond to the first and third percentile. Whiskers beyond these points denote $1.5 \times$ the interquartile range. $P$ values were determined by two-sided, Wilcoxon rank-sum test. 
A
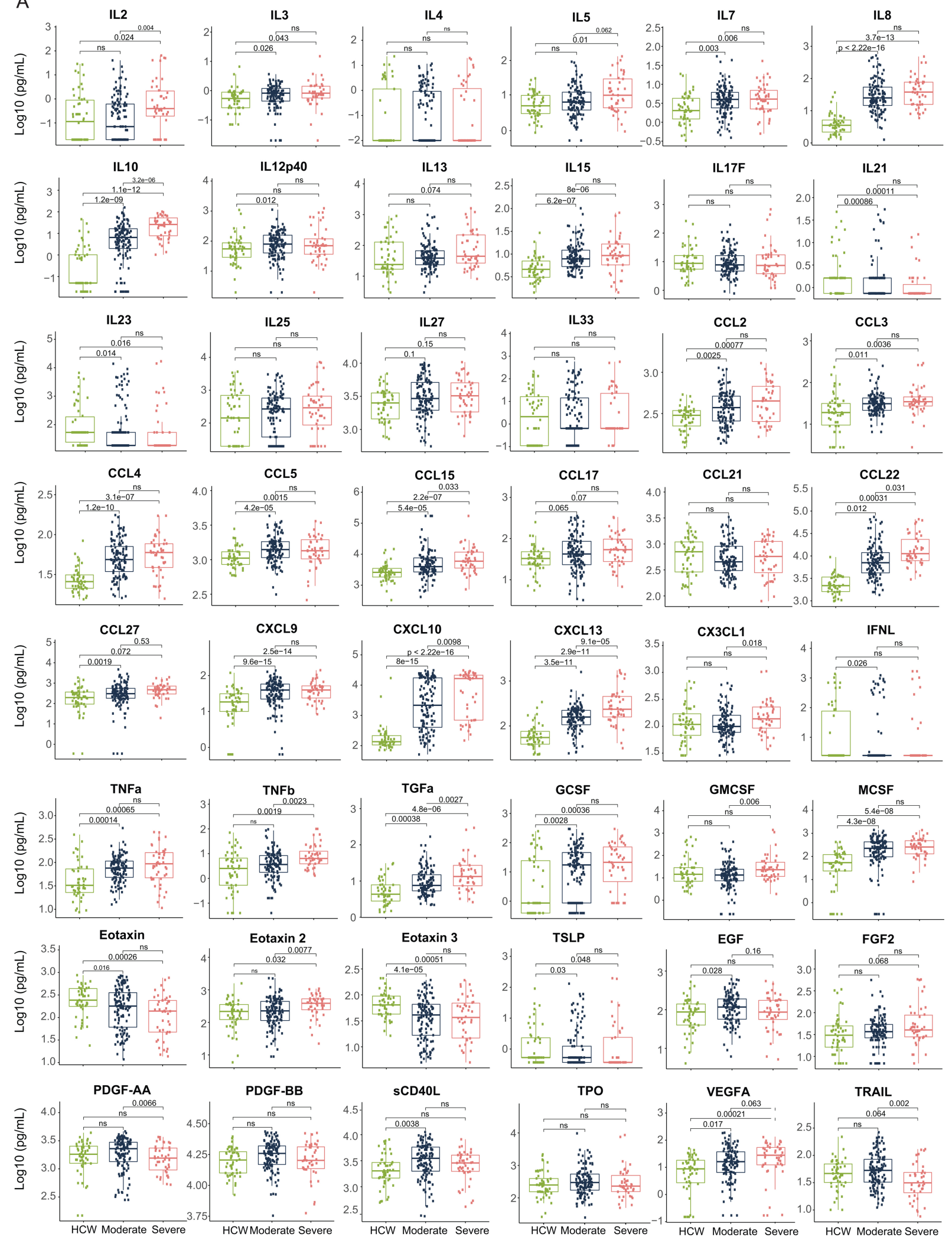

Extended Data Fig. 3 | Overview cytokine and chemokines profiles of COVID-19 patients. a, Quantification of cytokines in the periphery plotted as $\log _{10}$-transformed concentrations. Each dot represents a separate time point per subject (HCW, $n=47$; Moderate, $n=124$; Severe, $n=45$ ). For all boxplots, the centre is drawn through the median of the measurement, while the lower and upper bounds of the box correspond to the first and third percentile. Whiskers beyond these points denote $1.5 \times$ the interquartile range. $P$ values were determined by two-sided, Wilcoxon rank-sum test. 

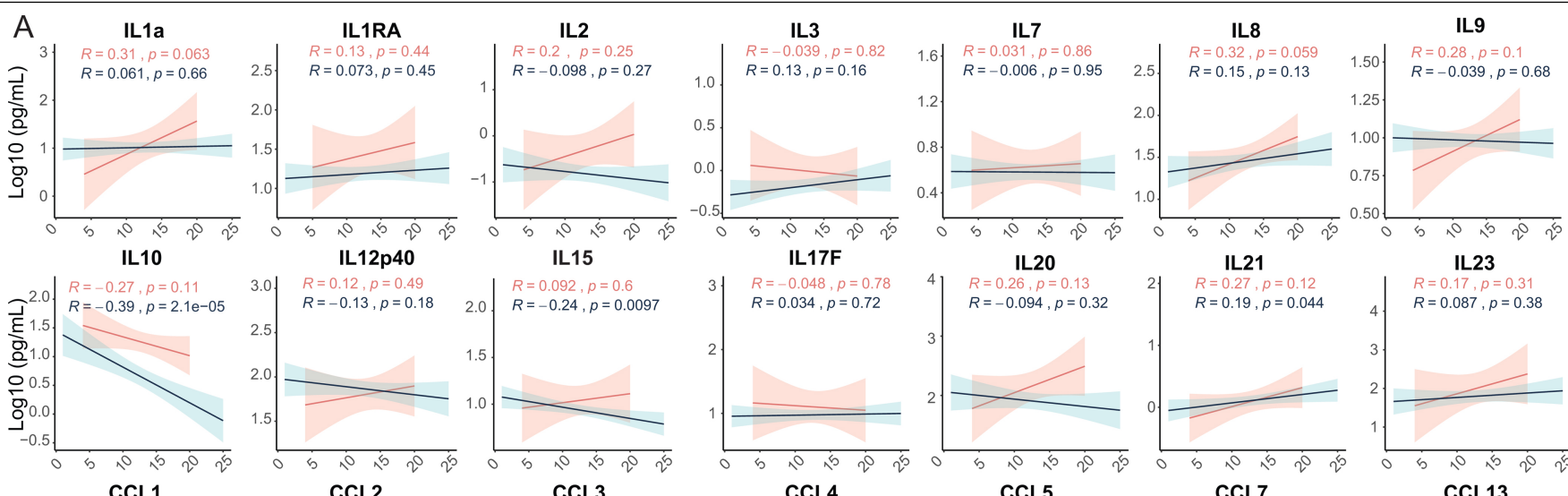

IL17F

IL21

IL23
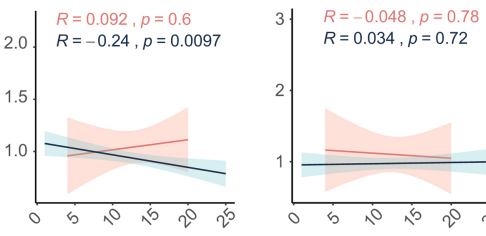

IL20
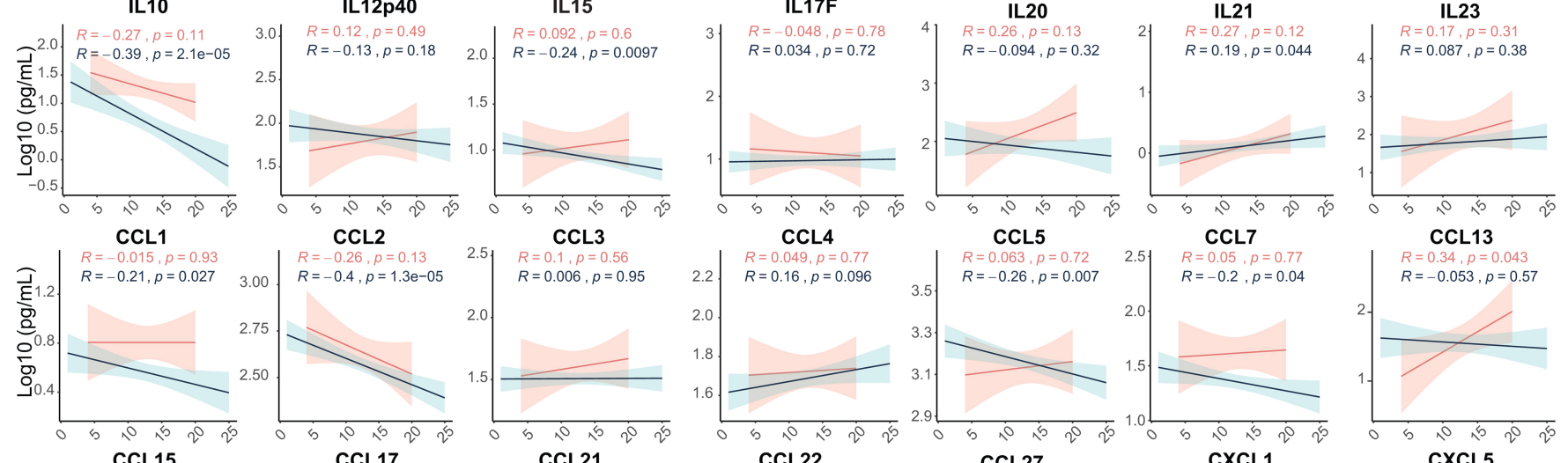

CCL3
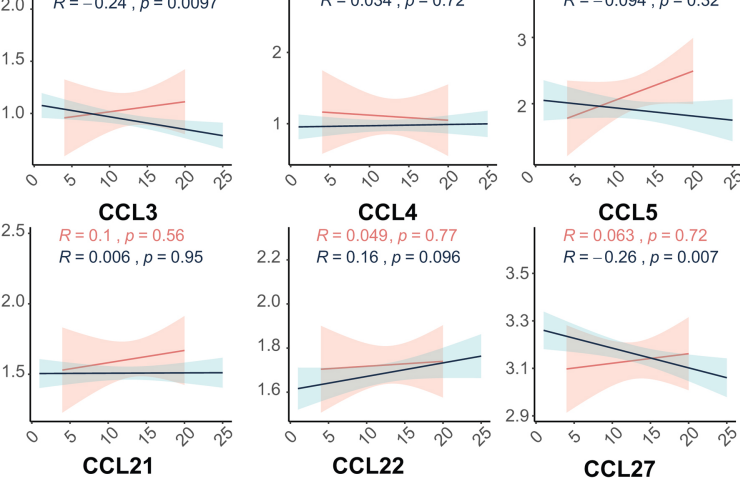

$R=0.05, p=0.77$
$R=-0.2, p=0.04$
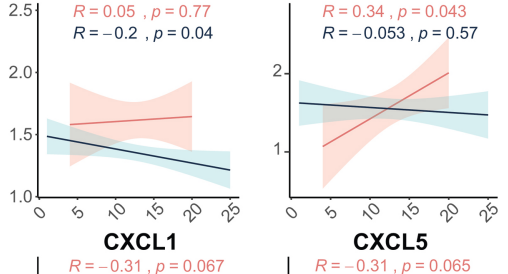

\section{CCL22}
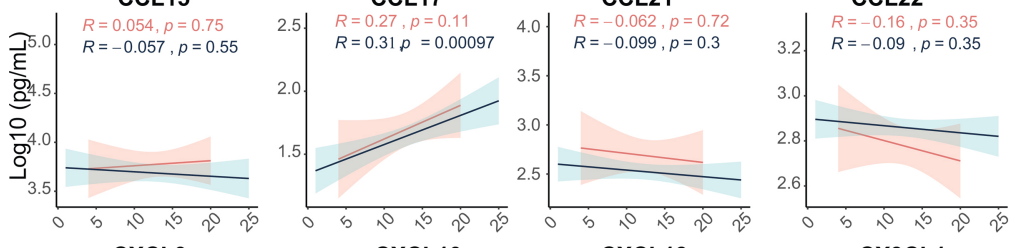

CCL27
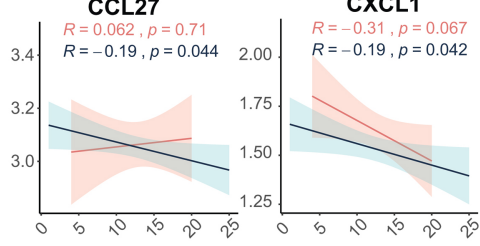

CXCL5

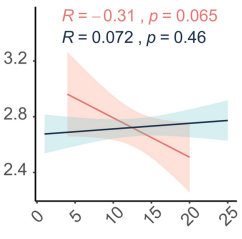

\section{CXCL13}
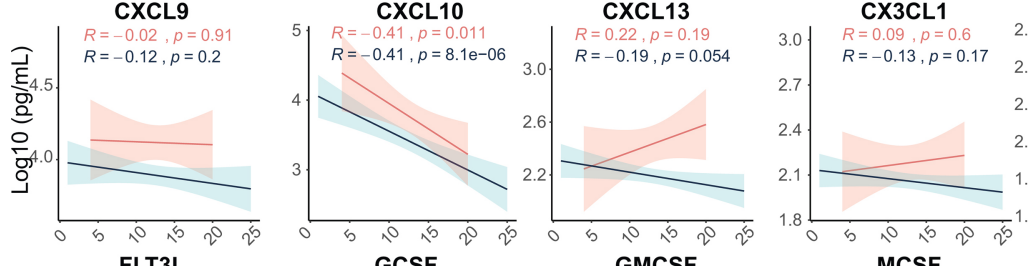

TNFa

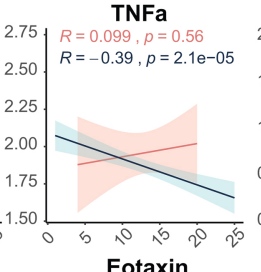

TNFb

TGFa
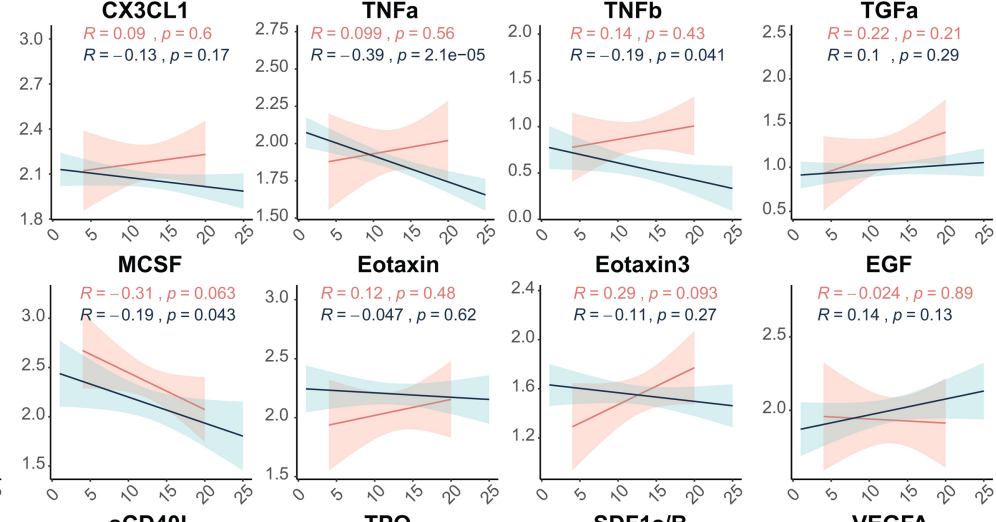

Eotaxin3
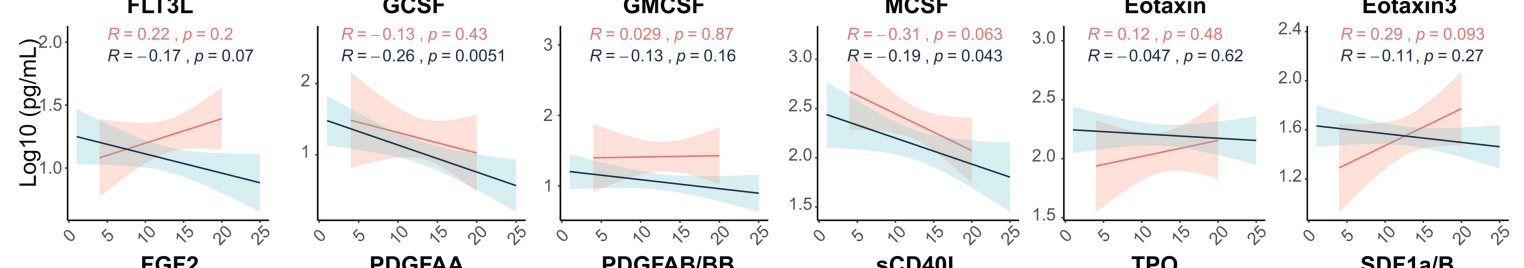

EGF

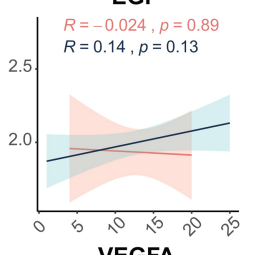

$=0.18, p=0.29$

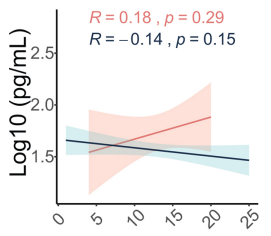

LIF
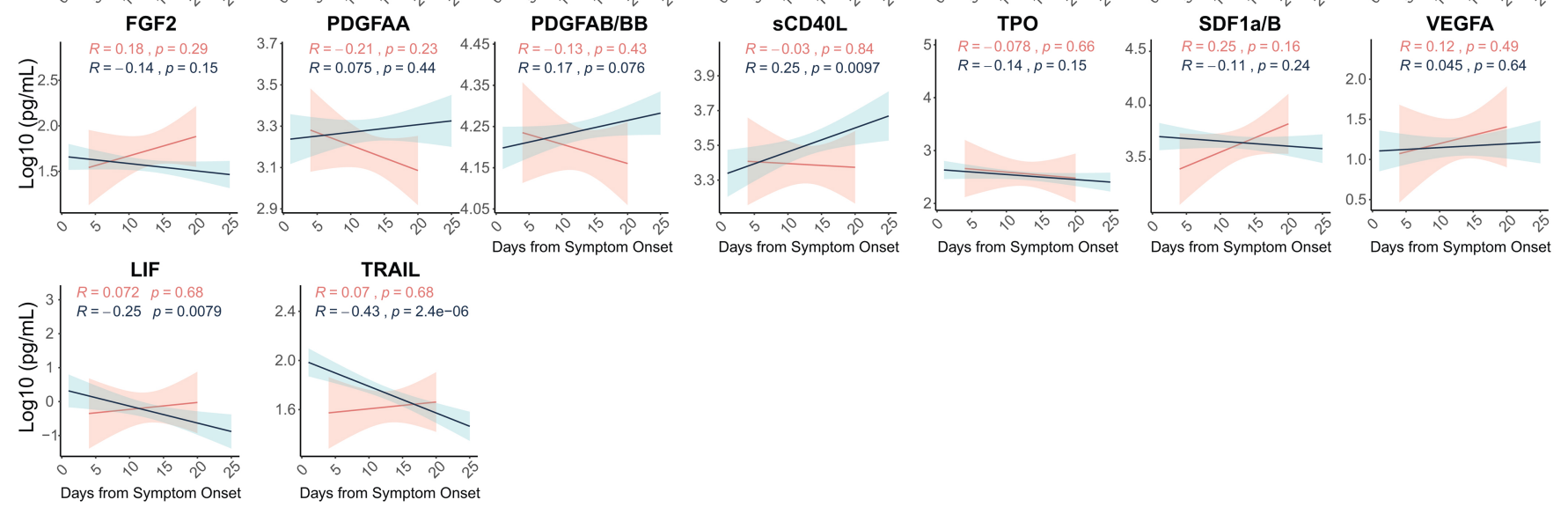

TRAIL

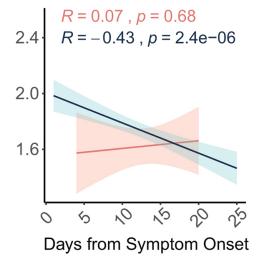

Extended Data Fig. 4 | Longitudinal cytokines and chemokines of COVID-19 patients. a, Quantification of cytokines plotted as $\log _{10}$-transformed concentration over time according to the days of symptom onset for patients with moderate disease $(n=112)$ or severe disease $(n=39)$. The dotted green line represents the mean measurement from uninfected HCWs. Regression lines

are indicated by the dark blue (moderate) or red (severe) solid lines. Associated, Pearson's correlation coefficients and linear regression significance are in pink (moderate) or dark blue (severe). 95\% confidence intervals for the regression lines are denoted by the pink (moderate) or dark blue (severe) filled areas. 
A
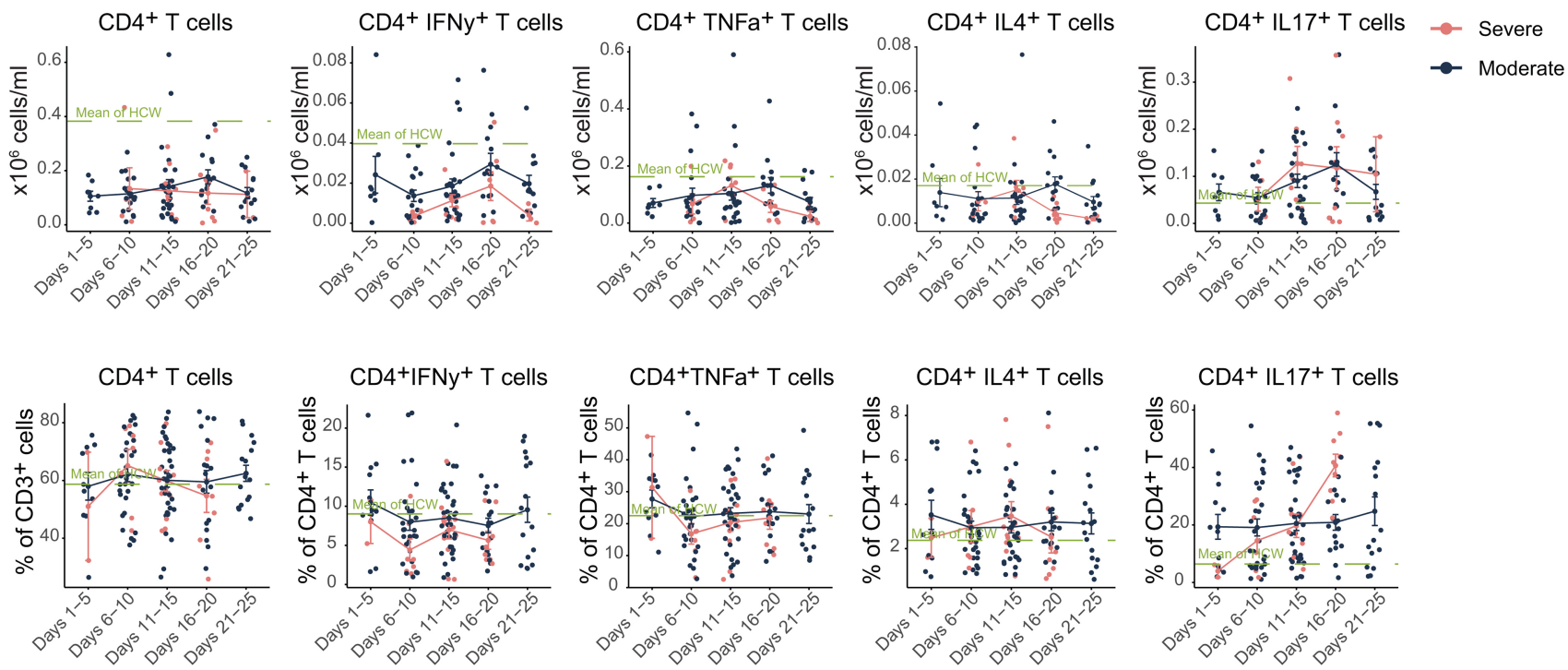

B
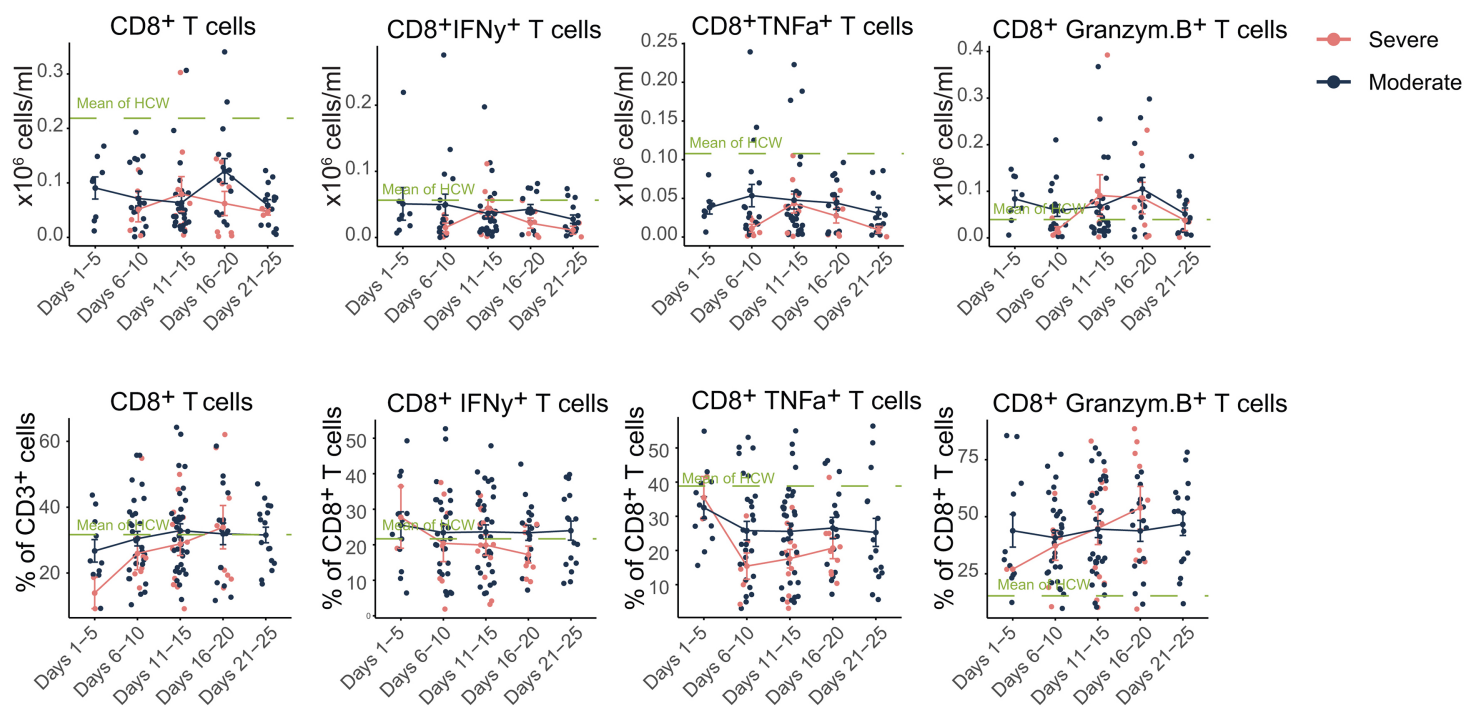

Extended Data Fig. $5 \mid \mathrm{T}$ cell immune profiles in moderate and severe patients. a, b, $\mathrm{CD}^{+}(\mathbf{a})$ and $\mathrm{CD}^{+}(\mathbf{b}) \mathrm{T}$ cell populations of interest, plotted as a percentage of parent populations, over time according to the days following symptom onset for patients with moderate disease $(n=118)$ or severe disease $(n=41)$. Each dot represents a distinct patient and time point arranged by intervals of five days until 25 days. Dark blue or pink lines pass through the mean of each measurement at the specified time interval; error bars at this intersection denote s.e.m. The dotted green line represents the mean measurement from uninfected HCWs. 

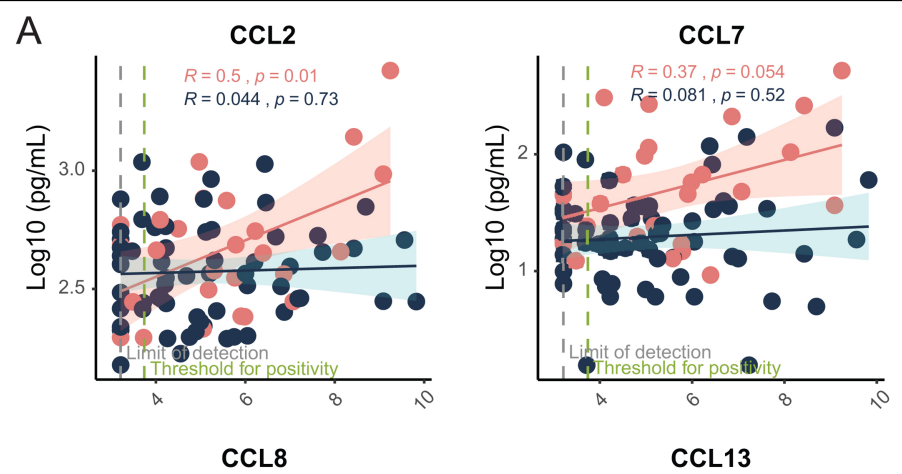

B Moderate (Non-ICU)
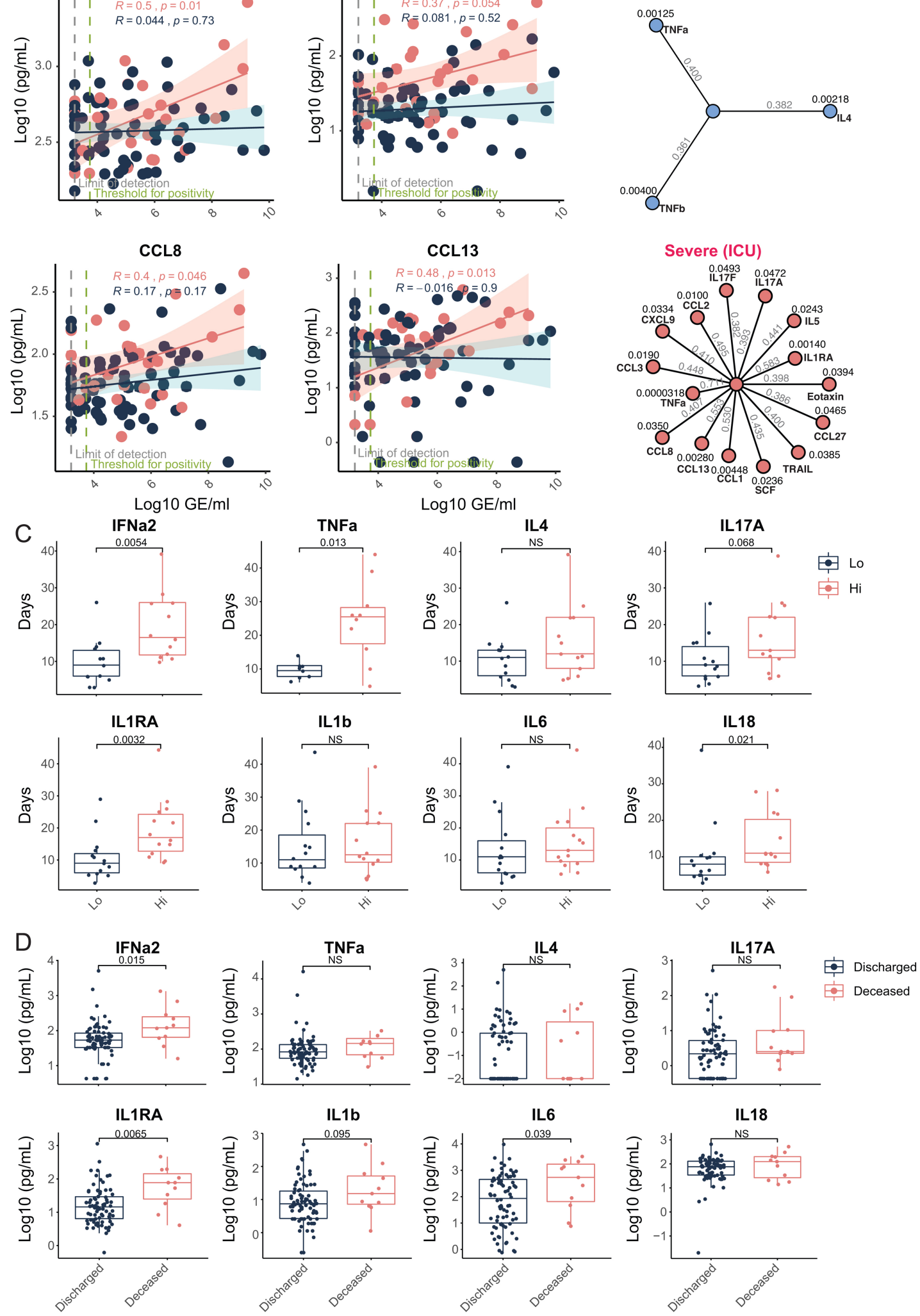

Extended Data Fig. 6 | See next page for caption. 


\section{Article}

Extended Data Fig. 6 |Early cytokine profile distinguishes moderate and severe outcomes. a, Quantification of $\log _{10}$-transformed cy tokine concentrations plotted continuously with NP viral load (expressed as $\log _{10}$ genomic equivalents $(\mathrm{GE}) / \mathrm{ml}$ ) per within an individual patient and time point. Regression lines are indicated by the dark blue (moderate) or red (severe) solid lines for patients with moderate disease $(n=112)$ or severe disease $(n=39)$, respectively. Associated Pearson's correlation coefficients, and linear regression significance are in pink (moderate) or dark blue (severe). 95\% confidence intervals for the regression lines are denoted by the pink (moderate) or dark blue (severe) filled areas.b, Correlation map of highly correlated cytokines with NP viral load in patients with moderate (blue) or severe disease (red). Pearson's correlation coefficients are indicated in grey, connecting the central node, NP viral load, with peripheral nodes; $P$ values for each correlation are indicated above each peripheral node. c, Length of hospital stay plotted per patient against an individual's baseline plasma cytokine measurements ( $<12$ days from symptom onset), which were grouped according to high or low expression ( $>0.5 \log _{10}$-transformed difference): IFNa2 (Hi:12, Lo:13), TNFa (Hi:6, Lo:4), IL4 (Hi:7, Lo:11), IL4 (Hi:8, Lo:6), IL1RA (Hi:8, Lo:7), IL1b (Hi:11, Lo:5), IL6 (Hi:8, Lo:7), IL18 (Hi:5, Lo:5). d, Baseline plasma cytokine measurements for each patient who was either discharged from the hospital $(n=83)$ or expired during treatment for COVID-19 $(n=11)$. For all boxplots, the centre is drawn through the median of the measurement, while the lower and upper bounds of the box correspond to the first and third percentile. Whiskers beyond these points denote $1.5 \times$ the interquartile range. $P$ values were determined by two-sided, Wilcoxon rank-sum test. 
A

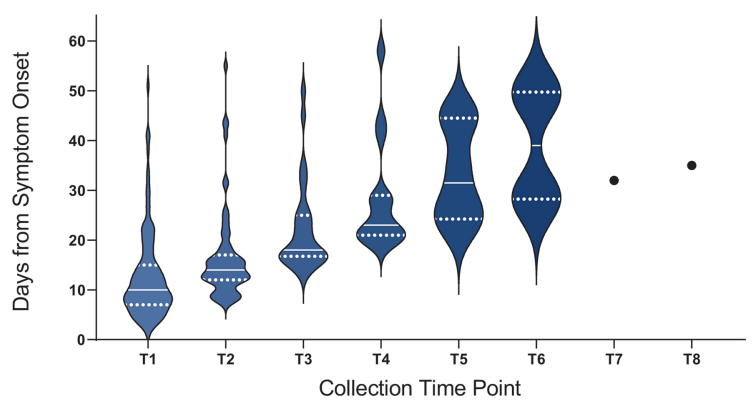

C

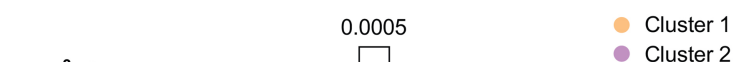

B

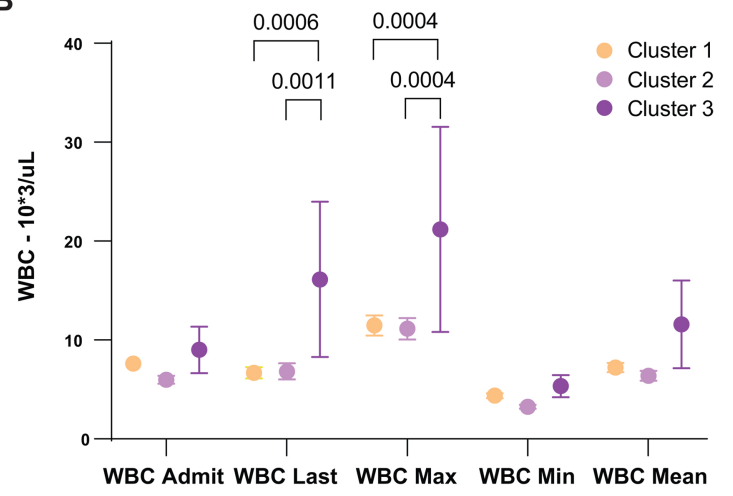

D

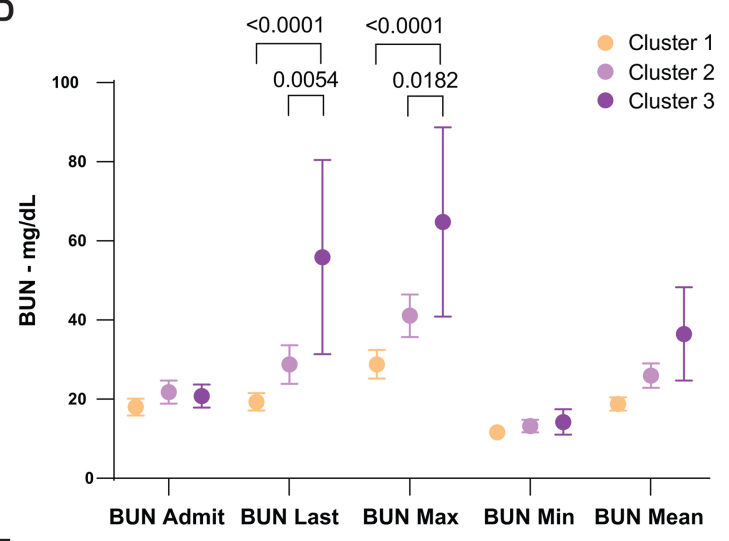

$\mathbf{F}$

Cluster 1

- Cluster 2

- Cluster 3

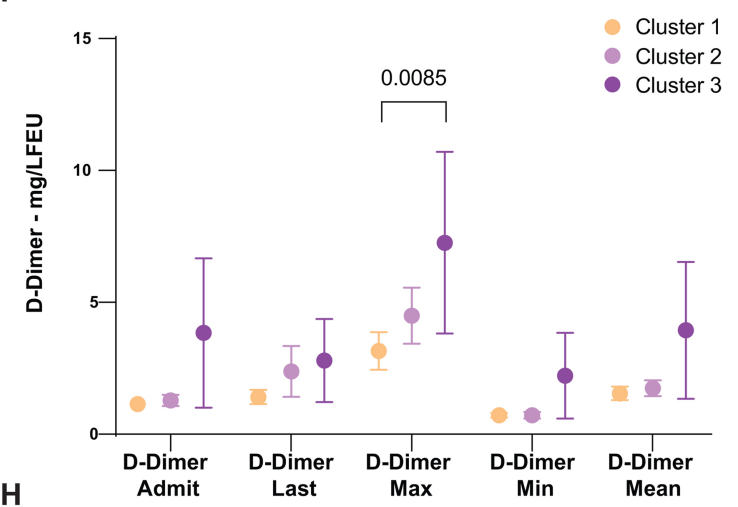

H

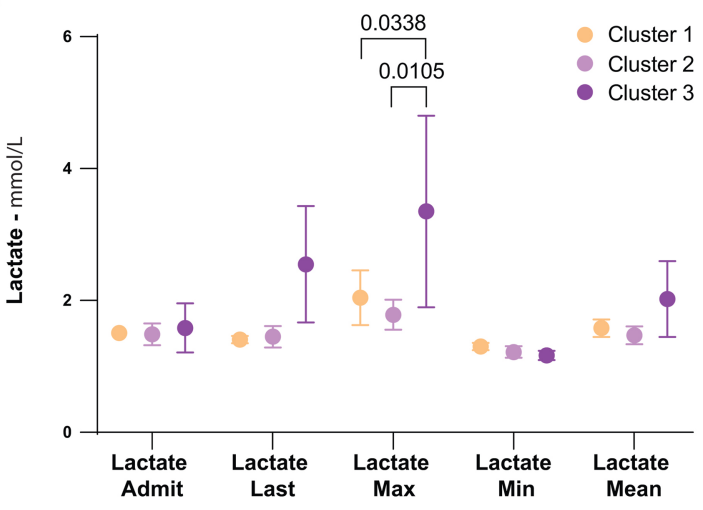

Extended Data Fig. 7 |See next page for caption. 


\section{Article}

Extended Data Fig. 7 | Distribution of days from symptom onset stratified by collection time point and select cluster clinical data. a, Correlation of days from symptom onset and samples collection time points. Violin plots comparing the distributions of days from symptom for each patient ordered by sequential IMPACT study time points (1-8). Study time points 7 and 8 are represented by discrete points for the single patient collected at each. Violin plots display median values (solid line) and associated quartiles (dashed lines) T1-8 (time point 1 to 8 ). b-h, Aggregated clinical data for patients in clusters 1-3. Displayed are laboratory values at time of admission to YNHH ("admit"); last recorded values from duration of admission ("last”); maximum recorded values from duration of admission ("max"); minimum recorded values from duration of admission ("min"); and average recorded values for duration of admission (“mean"). Scatter plots show cluster means with s.e.m. plotted above and below. Clusters were subsequently compared using ordinary twoway ANVOA and post hoc pairwise comparisons are identified where significant (adjusted $P$ values displayed, Tukey's method for multiple comparisons). 


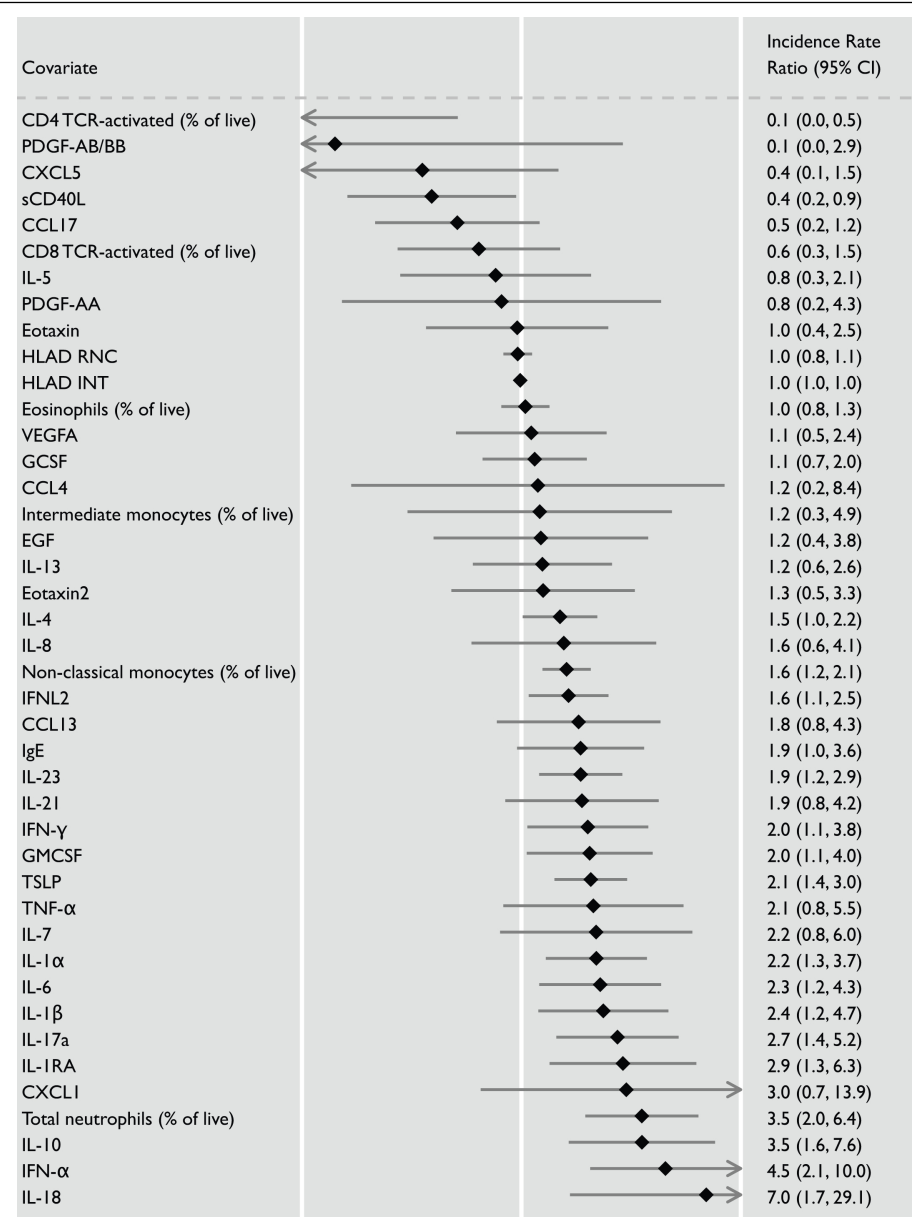

Extended Data Fig. 8 | Risk of death according to biomarkers levels. Forest plots comparing the risk of death among ill patients. Each effect estimate represents an individual regression estimate with a Poisson family, log link, and robust variance estimation; each model accounts for repeated measures within one individual through the use of generalized estimating equations (GEE).
Measurements are divided into three time-periods: 0-11 days after symptom onset, 12-19 days after symptom onset, and $\geq 20$ days after symptom onset. If an individual had more than one measurement of a biomarker during any particular time period, we used the average of all values. Each model controls for participant age and gender. 

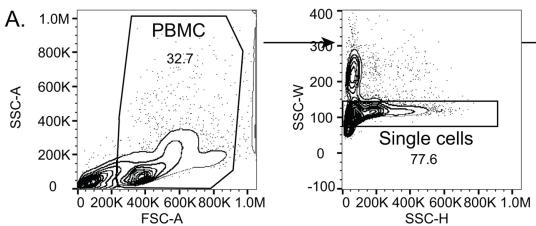

From $\mathrm{CD}^{-} \mathrm{CD} 19^{-}$
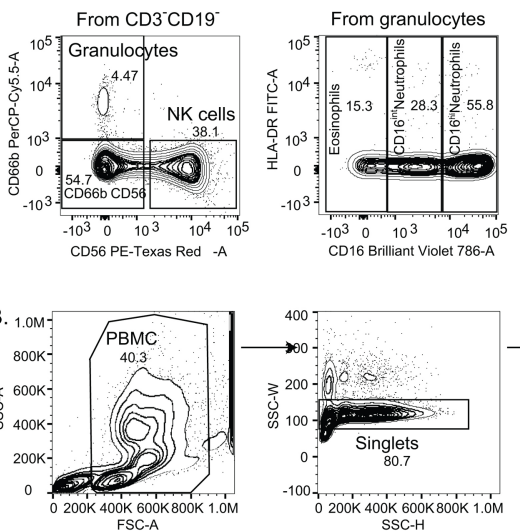

From $\mathrm{CD}^{+} \mathrm{T}$ cells
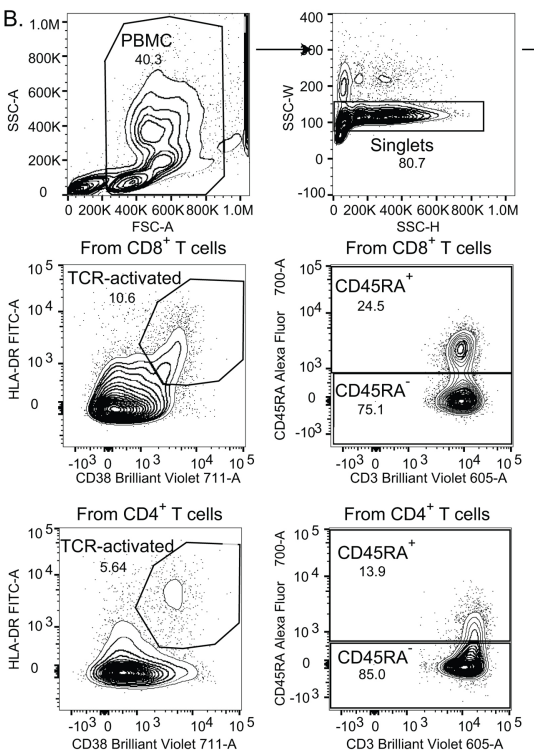

From $\mathrm{CD}^{+}{ }^{+} \mathrm{CD} 45 \mathrm{RA}^{-} \mathrm{T}$ cells
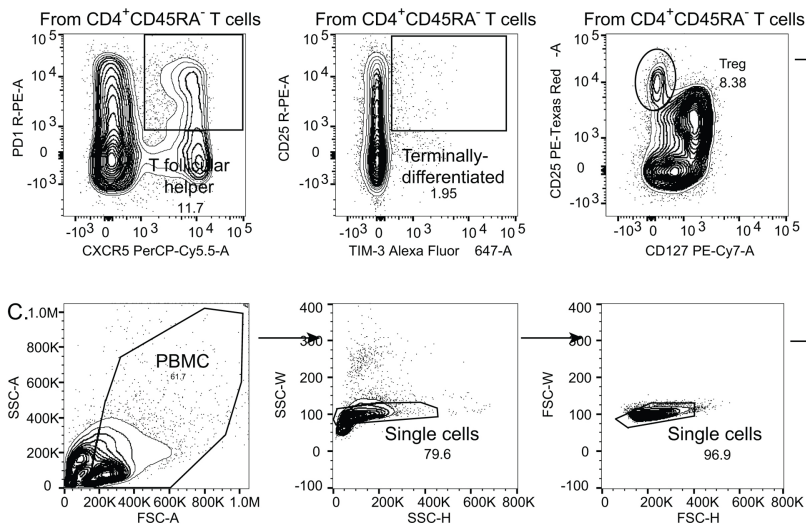

From $\mathrm{CD} 8^{+} \mathrm{T}$ cells
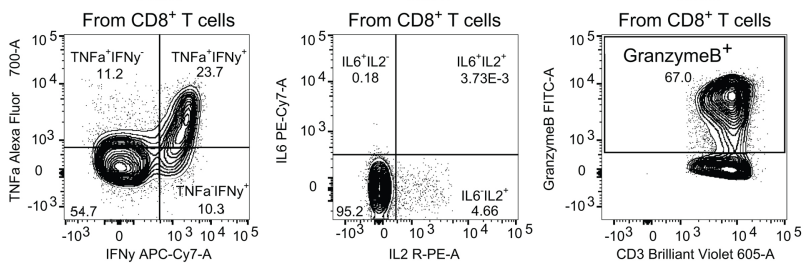

Extended Data Fig. 9 | Gating strategies. Gating strategies are shown for the key cell populations described in Figs. 1b, c, 2d-f, and in Extended Data Figures. a, Leukocyte gating strategy to identify lymphocytes, granulocytes, monocytes, pDCs, and cDCs in Figs. 1b, c, 2d-f and Extended Data Fig. 2a.b, T cell surface staining gating strategy to identify CD 4 and CD8 T cells, TCR-activated T cells,

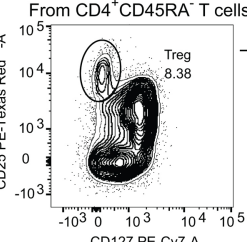

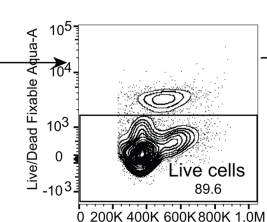

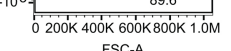

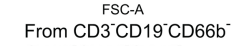

CD56 ${ }^{\circ} \mathrm{CD} 14^{-\mathrm{CD} 16}$
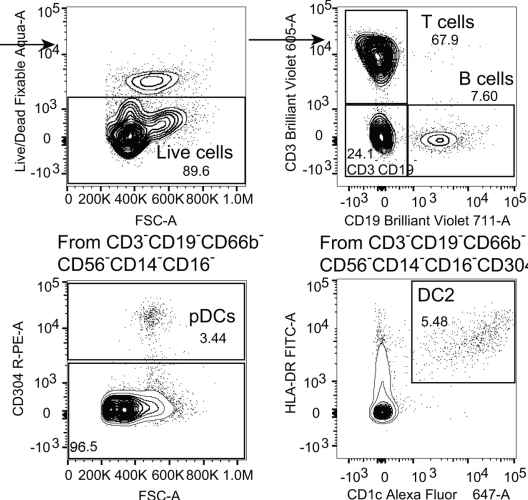

$\begin{array}{lll}10^{4} & 10 \\ & 10 & 0\end{array}$

From CD3 ${ }^{-C D} 19^{-} \mathrm{CD}^{-} \mathrm{b}^{-}$

CD56 ${ }^{-} \mathrm{CD} 14^{-} \mathrm{CD} 16^{-} \mathrm{CD} 304^{-}$
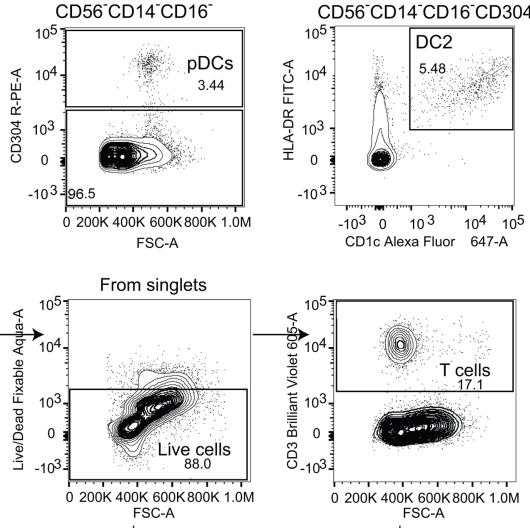

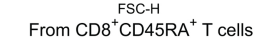

From $\mathrm{CD}^{+}{ }^{+} \mathrm{CD} 45 \mathrm{RA}^{-} \mathrm{T}$ cells

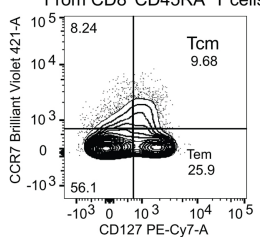

From CD8 ${ }^{+}$CD 45 RA- $T$ cells

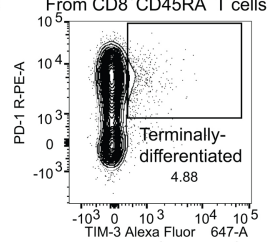

From $\mathrm{CD}^{+}{ }^{+} \mathrm{CD} 45 \mathrm{RA}^{+}$

From $\mathrm{CD} 4^{+} \mathrm{CD} 45 \mathrm{RA}^{+} \mathrm{T}$ cells
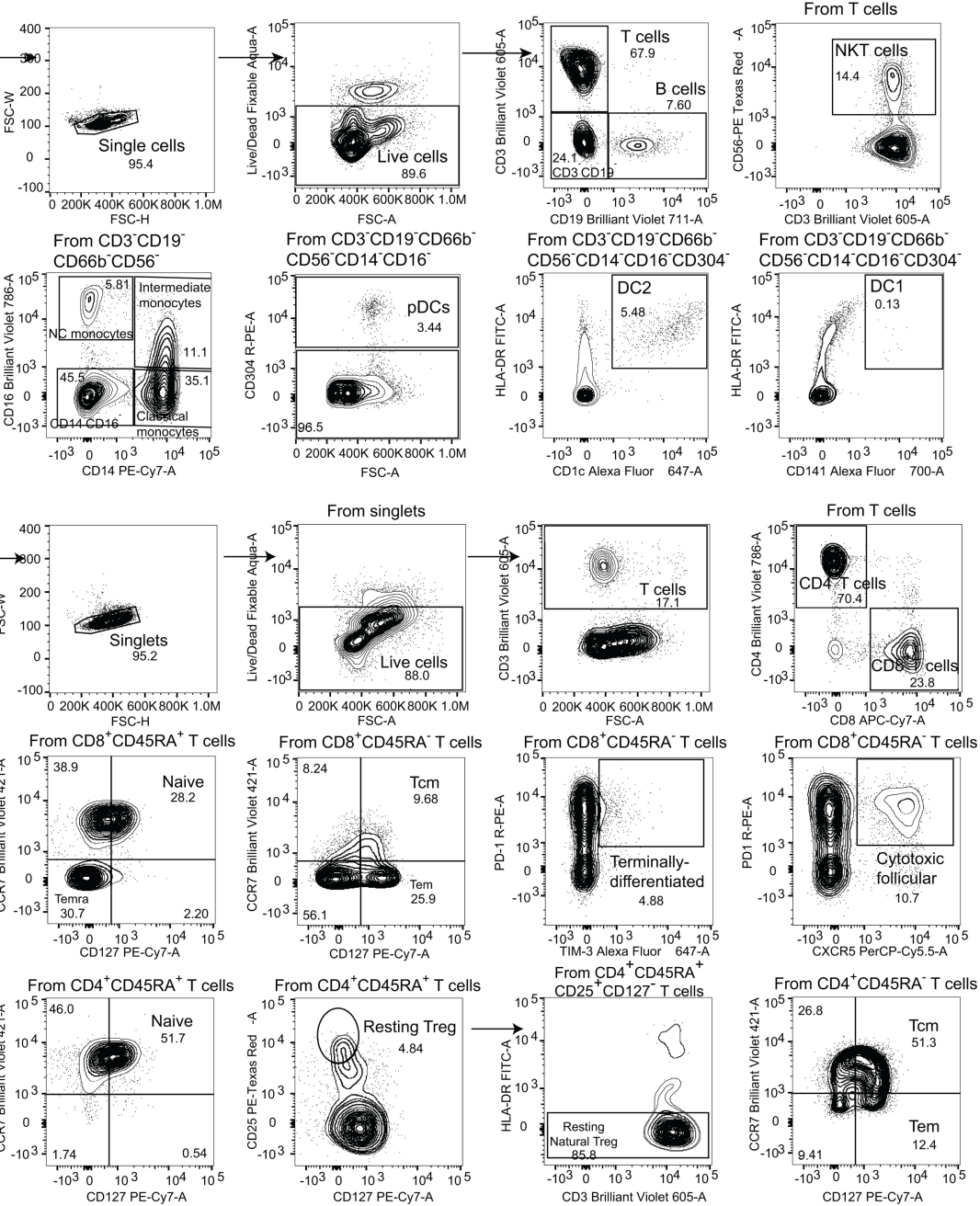
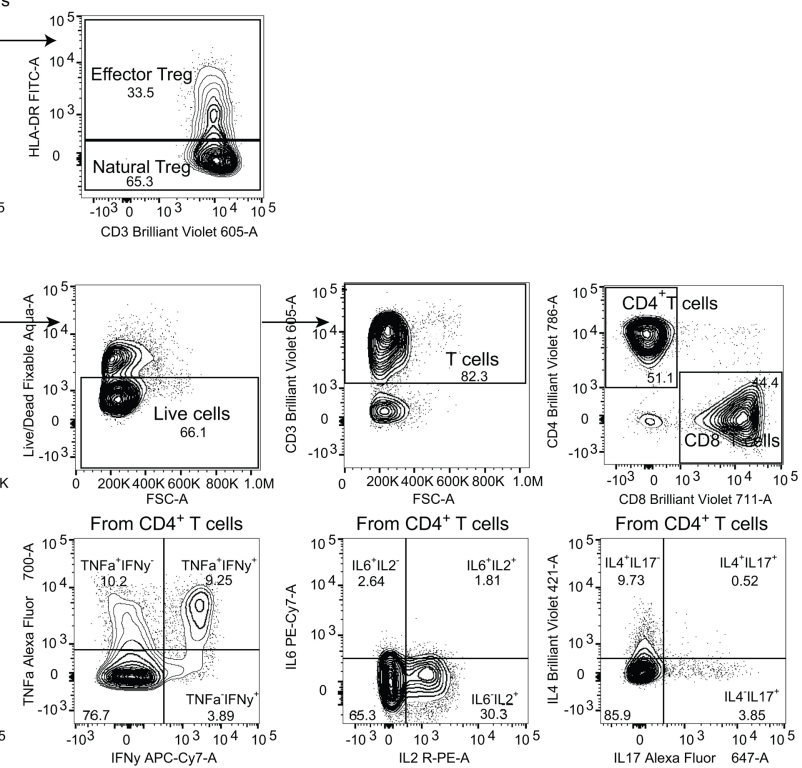

terminally-differentiated T cells, and additional subsets as shown in Extended Data Fig. 2b. c, Intracellular T cell gating strategy to identify CD4 and/or CD8 T cells secreting TNF, IFN $\gamma$, IL-6, IL-2, granzyme B, IL-4, and/or IL-17 in Extended Data Figs. 2c, 5a, b. 
Extended Data Table 1 | Basic demographics for IMPACT cohort

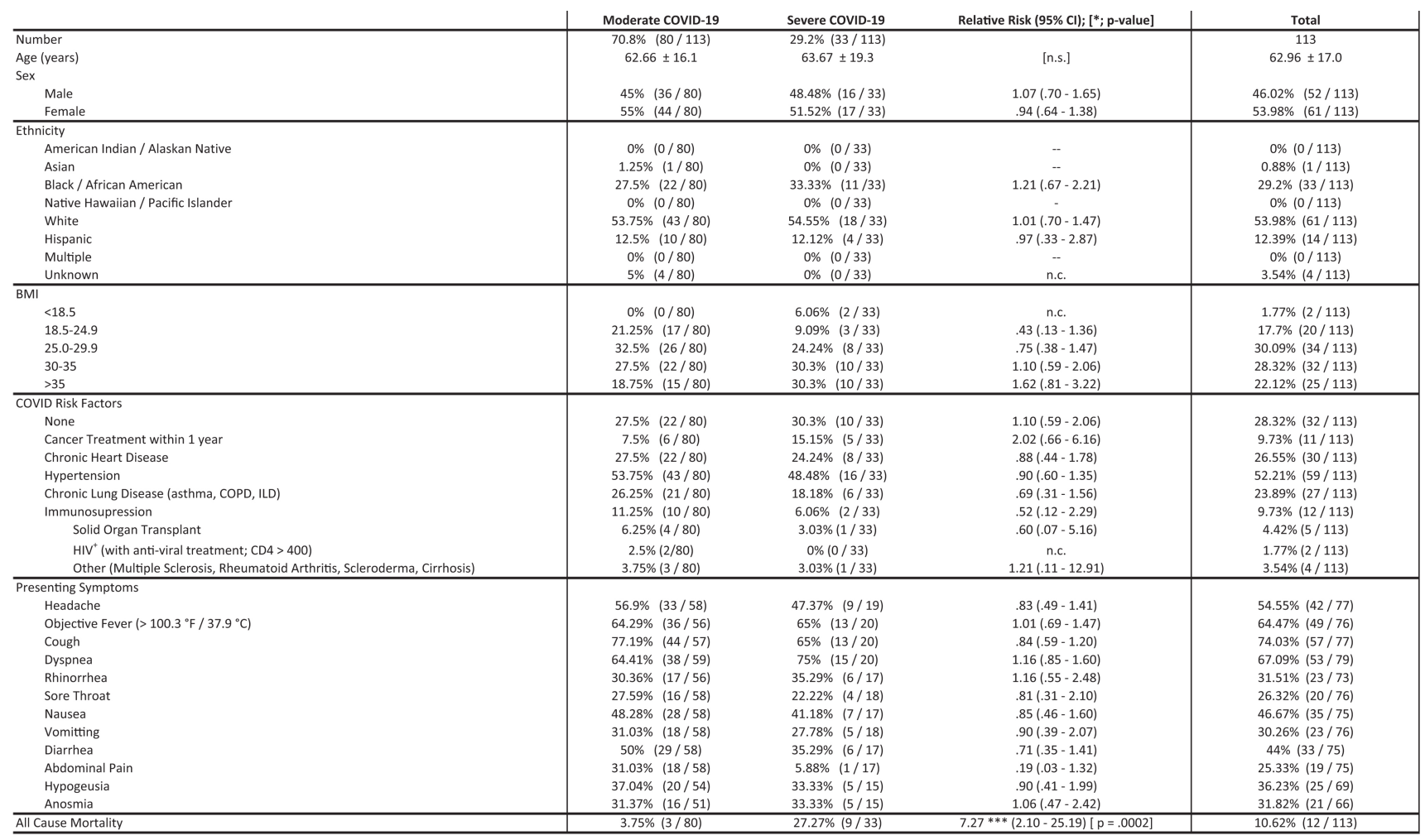

Unless otherwise noted, relative risks were not statistically significant. Moderate (clinical score 1-3) and severe (clinical score 4-5) disease status were assigned as described in Methods. Percentages of subgroup (moderate or severe) are shown for each category with respective counts in parenthesis. Average age was calculated with accompanying sample standard deviation. Ethnicity and BMI were extracted from most recent electronic medical record (EMR) data. Select COVID-19 risk factors were scored by a clinical infectious disease physician. Presenting simptoms were recorded through direct interview with patient or surrogate or retrospective EMR review. 


\section{Reporting Summary}

Nature Research wishes to improve the reproducibility of the work that we publish. This form provides structure for consistency and transparency in reporting. For further information on Nature Research policies, see our Editorial Policies and the Editorial Policy Checklist.

\section{Statistics}

For all statistical analyses, confirm that the following items are present in the figure legend, table legend, main text, or Methods section.

$\mathrm{n} / \mathrm{a} \mid$ Confirmed

$\square \bigotimes$ The exact sample size $(n)$ for each experimental group/condition, given as a discrete number and unit of measurement

Х $\square$ A statement on whether measurements were taken from distinct samples or whether the same sample was measured repeatedly

$\triangle$ The statistical test(s) used AND whether they are one- or two-sided

Only common tests should be described solely by name; describe more complex techniques in the Methods section.

$\bigotimes$ A description of all covariates tested

$\bigotimes$ A description of any assumptions or corrections, such as tests of normality and adjustment for multiple comparisons

$\triangle$ A full description of the statistical parameters including central tendency (e.g. means) or other basic estimates (e.g. regression coefficient)

AND variation (e.g. standard deviation) or associated estimates of uncertainty (e.g. confidence intervals)

$\varnothing$ For null hypothesis testing, the test statistic (e.g. $F, t, r$ ) with confidence intervals, effect sizes, degrees of freedom and $P$ value noted

Give P values as exact values whenever suitable.

$\bigotimes \square$ For Bayesian analysis, information on the choice of priors and Markov chain Monte Carlo settings

$\square \bigotimes$ For hierarchical and complex designs, identification of the appropriate level for tests and full reporting of outcomes

$\bigotimes$ Estimates of effect sizes (e.g. Cohen's $d$, Pearson's $r$ ), indicating how they were calculated

Our web collection on statistics for biologists contains articles on many of the points above.

\section{Software and code}

Policy information about availability of computer code

Data collection EPIC EHR software (retrospective EMR review and clinical data aggregation) and REDCap 9.3.6 (clinical data aggregation).

Data analysis GraphPad PRISM version 8.0.2 (statistics/graphics), R 3.4.3 (graphs/statistics), JMP15 (graphs), ggplot2, caret, tidyverse, ggpubr, Igraph, mlbench, and ggstatsplot, Flowjo software version 10.6 software (Tree Star).

For manuscripts utilizing custom algorithms or software that are central to the research but not yet described in published literature, software must be made available to editors and reviewers. We strongly encourage code deposition in a community repository (e.g. GitHub). See the Nature Research guidelines for submitting code \& software for further information.

\section{Data}

Policy information about availability of data

All manuscripts must include a data availability statement. This statement should provide the following information, where applicable:

- Accession codes, unique identifiers, or web links for publicly available datasets

- A list of figures that have associated raw data

- A description of any restrictions on data availability

The data generated during the current study will be available before publication in a public repository. Accession code number: SDY1655 


\section{Field-specific reporting}

Please select the one below that is the best fit for your research. If you are not sure, read the appropriate sections before making your selection.

\ Life sciences $\quad \square$ Behavioural \& social sciences $\quad \square$ Ecological, evolutionary \& environmental sciences

For a reference copy of the document with all sections, see nature.com/documents/nr-reporting-summary-flat.pdf

\section{Life sciences study design}

All studies must disclose on these points even when the disclosure is negative.

Sample size No statistical methods were used to calculate the sample size. Sample size was determined based on the number of patients admitted to YaleNew Haven Hospital (YNHH) between March 18th and May 5th that were enrolled and consented with th current study. This study enrolled 135 patients admitted to the Yale New Haven Health care network under IRB and HIC approved protocol \#2000027690. Patients were identified though screening of EMR records for potential enrollment. Informed consent was obtained by trained staff and sample collection commenced immediately upon study enrollment. Clinical specimens were collected approximately every 4 days where an individual's clinical status permitted, and was continued until patient discharge or expiration.

Data exclusions 135 COVID-19 patients were enrolled on this study however 22 were excluded. Those included: Pregnant women and patients on active chemotherapy. Specifically, cytokine ELISAs from two individuals were excluded from analysis due to poor sample quality. Measurements from these individuals were outliers (beyond $1.5 x$ the interquartile range) in more than half of the cytokines measured. This strongly suggested that a technical error occurred during these two experiments. Finally, for each individual boxplot, line graph, or linear regression, unique values that fell into the top or bottom $1 \%$ were excluded. Duplicate values within this range were not excluded. This applies only to unique values, such that two identical measurements falling into this range will remain in the analysis. We chose this very conservative method of exclusion in order to most faithfully represent the heterogeneity of our data, without allowing for extreme outliers to obscure our analyses. This is particularly true in situations in which we subset the data further by time intervals; with a smaller $n$ in each time interval, extreme outliers disproportionately skew the mean/median at this point. Finally for the health donors group, asymptomatic or presymptomatic healthcare workers were excluded (when positive for SARS-CoV2 q-RT-PCR or serology).

Replication The findings were not replicated - longitudinal analyses from human individuals.

Randomization Patients were stratified by disease severity (moderate and severe) based on based on oxygen levels and intensive care unit (ICU) requirement. Moderate disease status (Clinical Score 1, 2 and 3) was defined as: (1) SARS-CoV-2 infection requiring hospitalization without supplemental oxygen, (2) infection requiring non-invasive supplemental oxygen ( $<3 \mathrm{~L} / \mathrm{min}$, sufficient to maintain greater than $92 \% \mathrm{SpO} 2)$, (3) infection requiring non-invasive supplemental oxygen ( $>3 \mathrm{~L}$ supplemental oxygen to maintain $\mathrm{SpO} 2>92 \%$, or, required $>2 \mathrm{~L}$ supplemental oxygen to maintain SpO2 > 92\% and had a high sensitivity C-reactive protein (CRP) > 70) and received tocilizumab. Severe disease status (Clinical score 4 and 5) was defined as infection meeting all criteria for clinical score 3 while also requiring admission to the YNHH Intensive Care Unit (ICU) and $>6 \mathrm{~L}$ supplemental oxygen to maintain $\mathrm{SpO} 2$ > 92\% (4); or infection requiring invasive mechanical ventilation / extracorporeal membrane oxygenation (ECMO) in addition to glucocorticoid / vasopressor administration (5). Clinical score 6 was assigned for deceased patients.

At the time of sample acquisition and processing, scientists were completely unaware of the patients' conditions. Blood acquisition is performed and recorded by a separate team. Information of patients' conditions are not available until after processing and analysing raw data by flow cytometry and ELISA. A clinical team, separate from the experimental team, performs chart review to determine patients' relevant statistics. Cytokines and facs analyses were blinded. Patients clinical information and clinical scores coding were only revealed after data collection.

\section{Reporting for specific materials, systems and methods}

We require information from authors about some types of materials, experimental systems and methods used in many studies. Here, indicate whether each material, system or method listed is relevant to your study. If you are not sure if a list item applies to your research, read the appropriate section before selecting a response.

Materials \& experimental systems

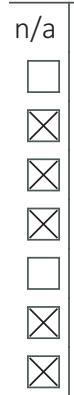

n/a Involved in the study

$\bigotimes$ Antibodies

Х Eukaryotic cell lines

Х $\square$ Palaeontology and archaeology

Х

$\square$

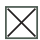

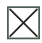

\begin{tabular}{l|l}
\multicolumn{2}{l}{ Methods } \\
\hline n/a & Involved in the study \\
\hline & $\square$ ChIP-seq \\
$\square$ & $\square$ Flow cytometry \\
$\square$ & $\square$ MRI-based neuroimaging
\end{tabular}



hCD16 (3G8) (1:100) (BioLegend), PE-Cy7 anti-hCD14 (HCD14) (1:300) (BioLegend), BV605 anti-hCD3 (UCHT1) (1:300) (BioLegend), BV711 anti-hCD19 (SJ25C1) (1:300) (BD Biosciences), AlexaFluor647 anti-hCD1c (L161) (1:150) (BioLegend), Biotin anti-hCD141 (M80) (1:150) (BioLegend), PE-Dazzle594 anti-hCD56 (HCD56) (1:300) (BioLegend), PE anti-hCD304 (12C2) (1:300) (BioLegend), APCFire750 anti-hCD11b (ICRF44) (1:100) (BioLegend), PerCP/Cy5.5 anti-hCD66b (G10F5) (1:200) (BD Biosciences), BV785 anti-hCD4 (SK3) (1:200) (BioLegend), APCFire750 or PE-Cy7 or BV711 anti-hCD8 (SK1) (1:200) (BioLegend), BV421 anti-hCCR7 (G043H7) (1:50) (BioLegend), AlexaFluor 700 anti-hCD45RA (HI100) (1:200) (BD Biosciences), PE anti-hPD1 (EH12.2H7) (1:200) (BioLegend), APC anti-hTIM3 (F38-2E2) (1:50) (BioLegend), BV711 anti-hCD38 (HIT2) (1:200) (BioLegend), BB700 anti-hCXCR5 (RF8B2) (1:50) (BD Biosciences), PECy7 anti-hCD127 (HIL-7R-M21) (1:50) (BioLegend), PE-CF594 anti-hCD25 (BC96) (1:200) (BD Biosciences), BV711 anti-hCD127 (HIL-7R-M21) (1:50) (BD Biosciences), BV421 anti-hIL17a (N49-653) (1:100) (BD Biosciences), AlexaFluor 700 anti-hTNFa (MAb11) (1:100) (BioLegend), PE or APC/Fire750 anti-hIFNy (4S.B3) (1:60) (BioLegend), FITC anti-hGranzymeB (GB11) (1:200) (BioLegend), AlexaFluor 647 anti-hIL-4 (8D4-8) (1:100) (BioLegend), BB700 anti-hCD183/CXCR3 (1C6/CXCR3) (1:100) (BD Biosciences), PE-Cy7 antihIL-6 (MQ2-13A5) (1:50) (BioLegend), PE anti-hIL-2 (5344.111) (1:50) (BD Biosciences), BV785 anti-hCD19 (SJ25C1) (1:300) (BioLegend), BV421 anti-hCD138 (MI15) (1:300) (BioLegend), AlexaFluor700 anti-hCD20 (2H7) (1:200) (BioLegend), AlexaFluor 647 anti-hCD27 (M-T271) (1:350) (BioLegend), PE/Dazzle594 anti-hlgD (IA6-2) (1:400) (BioLegend), PE-Cy7 anti-hCD86 (IT2.2) (1:100) (BioLegend), APC/Fire750 anti-hlgM (MHM-88) (1:250) (BioLegend), BV605 anti-hCD24 (ML5) (1:200) (BioLegend), BV421 anti-hCD10 (HI10a) (1:200) (BioLegend), BV421 anti-CDh15 (SSEA-1) (1:200) (BioLegend), AlexaFluor 700 Streptavidin (1:300) (ThermoFisher), BV605 Streptavidin (1:300) (BioLegend).

Validation

All antibodies used in this study are commercially available, and all have been validated by the manufacturers and used by other publications. Likewise, we titrated these antibodies according to our own our staining conditions. The following were validated in the following species: BB515 anti-hHLA-DR (G46-6) (BD Biosciences) (Human, Rhesus, Cynomolgus, Baboon), BV785 anti-hCD16 (3G8) (BioLegend) (Human, African Green, Baboon, Capuchin Monkey, Chimpanzee, Cynomolgus, Marmoset, Pigtailed Macaque, Rhesus, Sooty Mangabey, Squirrel Monkey), PE-Cy7 anti-hCD14 (HCD14) (BioLegend) (Human), BV605 anti-hCD3 (UCHT1) (BioLegend) (Human, Chimpanzee), BV711 anti-hCD19 (SJ25C1) (BD Biosciences) (Human), AlexaFluor647 anti-hCD1c (L161) (BioLegend) (Human, African Green, Baboon, Cynomolgus, Rhesus), Biotin anti-hCD141 (M80) (BioLegend) (Human, African Green, Baboon), PE-Dazzle594 anti-hCD56 (HCD56) (BioLegend) (Human, African Green, Baboon, Cynomolgus, Rhesus), PE anti-hCD304 (12C2) (BioLegend) (Human), APCFire750 anti-hCD11b (ICRF44) (BioLegend) (Human, African Green, Baboon, Chimpanzee, Common Marmoset, Cynomolgus, Rhesus, Swine), PerCP/Cy5.5 anti-hCD66b (G10F5) (BD Biosciences) (Human), BV785 anti-hCD4 (SK3) (BioLegend) (Human), APCFire750 or PE-Cy7 or BV711 anti-hCD8 (SK1) (BioLegend) (Human, Cross-Reactivity: African Green, Chimpanzee, Cynomolgus, Pigtailed Macaque, Rhesus, Sooty Mangabey), BV421 anti-hCCR7 (G043H7) (BioLegend) (Human, African Green, Baboon, Cynomolgus, Rhesus), AlexaFluor 700 anti-hCD45RA (HI100) (BD Biosciences) (Human), PE anti-hPD1 (EH12.2H7) (BioLegend) (Human, African Green, Baboon, Chimpanzee, Common Marmoset, Cynomolgus, Rhesus, Squirrel Monkey), APC antihTIM3 (F38-2E2) (BioLegend) (Human), BV711 anti-hCD38 (HIT2) (BioLegend) (Human, Chimpanzee, Horse), BB700 anti-hCXCR5 (RF8B2) (BD Biosciences) (Human), PE-Cy7 anti-hCD127 (HIL-7R-M21) (BioLegend) (Human), PE-CF594 anti-hCD25 (BC96) (BD Biosciences) (Human, Rhesus, Cynomolgus, Baboon), BV711 anti-hCD127 (HIL-7R-M21) (BD Biosciences) (Human), BV421 anti-hIL-17a (N49-653) (BD Biosciences) (Human), AlexaFluor 700 anti-hTNFa (MAb11) (BioLegend) (Human, Cat, Cross-Reactivity: Chimpanzee, Baboon, Cynomolgus, Rhesus, Pigtailed Macaque, Sooty Mangabey, Swine), PE or APC/Fire750 anti-hIFNy (4S.B3) (BioLegend) (Human, Cross-Reactivity: Chimpanzee, Baboon, Cynomolgus, Rhesus), FITC anti-hGranzymeB (GB11) (BioLegend) (Human, Mouse, Cross-Reactivity: Rat), AlexaFluor 647 anti-hIL-4 (8D4-8) (BioLegend) (Human, Cross-Reactivity: Chimpanzee, Baboon, Cynomolgus, Rhesus), BB700 anti-hCD183/CXCR3 (1C6/CXCR3) (BD Biosciences) (Human, Rhesus, Cynomolgus, Baboon), PE-Cy7 anti-IL-6 (MQ2-13A5) (BioLegend) (Human), PE anti-hIL-2 (5344.111) (BD Biosciences) (Human), BV785 anti-hCD19 (SJ25C1) (BioLegend) (Human), BV421 anti-hCD138 (MI15) (BioLegend) (Human), AlexaFluor700 anti-hCD20 (2H7) (BioLegend) (Human, Baboon, Capuchin Monkey, Chimpanzee, Cynomolgus, Pigtailed Macaque, Rhesus, Squirrel Monkey), AlexaFluor 647 anti-hCD27 (M-T271) (BioLegend) (Human, Cross-Reacitivity: Baboon, Cynomolgus, Rhesus), PE/Dazzle594 anti-hlgD (IA6-2) (BioLegend) (Human), PE-Cy7 anti-hCD86 (IT2.2) (BioLegend) (Human, African Green, Baboon, Capuchin Monkey, Common Marmoset, Cotton-topped Tamarin, Chimpanzee, Cynomolgus, Rhesus), APC/Fire750 anti-hlgM (MHM-88) (BioLegend) (Human, African Green, Baboon, Cynomolgus, Rhesus), BV605 anti-hCD24 (ML5) (BioLegend) (Human, Cross-Reactivity: Chimpanzee), BV421 anti-hCD10 (HI10a) (BioLegend) (Human, African Green, Baboon, Capuchin monkey, Chimpanzee, Cynomolgus, Rhesus), BV421 anti-hCD15 (SSEA-1) (BioLegend) (Human), AlexaFluor 700 Streptavidin (1:300) (ThermoFisher), BV605 Streptavidin (1:300) (BioLegend).

\section{Human research participants}

\section{Policy information about studies involving human research participants}

Population characteristics

Recruitment

Ethics oversight
Cohort characteristics: age (62.96 \pm 17.0$)$, sex (Male 46.02\% / Females 53.98\%, Ethnicity (American Indian -Alaskan Native 0\%/ Asian (0.88\%) / Black -African American (29.2\%)/ Native Hawaiian-Pacific Islander(0\%)/ White (53.98\%)/ Hispanic (12.39\%). Full demographic data is included in Extended data table 1.

Patients admitted to the Yale New Haven Hospital (YNHH) between the 18th of March through the 27th of May 2020, were recruited to the Yale IMPACT study (Implementing Medical and Public Health Action Against Coronavirus CT) after testing positive for SARS-CoV2 by qRT-PCR. (serology was further confirmed for all patients enrolled). Patients were identified though screening of EMR records for potential enrollment with no self selection. Informed consent was obtained by trained staff and sample collection commenced immediately upon study enrollment. Clinical specimens were collected approximately every 4 days where an individual's clinical status permitted, and was continued until patient discharge or expiration.

Yale Human Research Protection Program Institutional Review Boards. Informed consents were obtained from all enrolled patients and healthcare workers. - Our research protocol was reviewed and approved by the Yale School of Medicine IRB and HIC (\#2000027690). Informed consent was obtained by trained staff and records maintained in our research database for the duration of our study. There were no minors included on this study. 


\section{Flow Cytometry}

\section{Plots}

Confirm that:

$\bigotimes$ The axis labels state the marker and fluorochrome used (e.g. CD4-FITC).

Х The axis scales are clearly visible. Include numbers along axes only for bottom left plot of group (a 'group' is an analysis of identical markers).

\All plots are contour plots with outliers or pseudocolor plots.

Х A numerical value for number of cells or percentage (with statistics) is provided.

\section{Methodology}

Sample preparation

Instrument

Software

Cell population abundance

Gating strategy
Freshly isolated PBMCs were stained for live and dead markers, blocked with Human TruStan FcX, stained for surface markers and then fixed with PFA 4\%. For intracellular cytokine staining following stimulation, cells were surface stained, washed and fixed in 4\% PFA. After permeabilization with 1X Permeabilization Buffer cells were stained for intracellular cytokines analysis.

Cells were acquired on an Attune NXT (ThermoFisher).

Data were analysed using FlowJo software version 10.6 software (Tree Star). n

Cell population abundance: Cells populations were reported in various formats including as a number or concentration of the patient's blood sample ( $\times 106$ cells $/ \mathrm{mL}$ ), as a proportion of live, single PBMC (\% of Live), or as a proportion of a parent gate (\% of CD4 T cells, \% of Monocytes, etc.). The full gating path for clarification is included in the extended figures.

SSC-A and FSC-A parameters were used to select leukocytes from isolated PBMCs. Live and dead cells were defined based on aqua staining. Singlets were separated based on SSC/ FSC parameters. Leukocytes were gated based on to identify lymphocytes (CD3/CD4/CD8/CD19/CD56 markers), granulocytes (CD16,CD14, HLA-DR markers) and pDCs, and cDCs (CD304, CD1c, CD141). TCR-activated T cells, Terminally-differentiated T cells, and additional subsets.were defined using HLA-DR, CD38, CCR7,CD127, PD1, TIM-3, CXCR5, CD45RA, CD25. Intracellular T cell gating strategy to identify CD4 and/or CD8 T cells secreting TNFa, IFN-y, IL-6, IL-2, GranzymeB, IL-4, and/or IL-17 were defined using the specif markers: CD3, CD4, CD8, TNF, IFN, IL-6, IL-2, IL-4, IL-17 and granzyme B.

$\bigotimes$ Tick this box to confirm that a figure exemplifying the gating strategy is provided in the Supplementary Information. 